\title{
Pseudo-Three-Stage Miller Op-Amp With Enhanced Small-Signal and Large-Signal Performance
}

\author{
Anindita Paul ${ }^{\circledR}$, Member, IEEE, Jaime Ramírez-Angulo ${ }^{\circledR}$, Fellow, IEEE, \\ Antonio J. López-Martín ${ }^{\circledR}$, Senior Member, IEEE, Ramón Gonzalez Carvajal, Senior Member, IEEE, \\ and José Miguel Rocha-Pérez, Member, IEEE
}

\begin{abstract}
A simple technique to implement highly power efficient class AB-AB Miller op-amps is presented in this paper. It uses a composite input stage with resistive local common mode feedback that provides class $A B$ operation to the input stage and essentially enhances the op-amp's effective transconductance gain, the dc open-loop gain, the gain-bandwidth product, and slew rate with just moderate increase in power dissipation. The experimental results of op-amps in strong inversion and subthreshold fabricated in a 130-nm standard CMOS technology validate the proposed approach. The op-amp has $9 \mathrm{~V} \cdot \mathrm{pF} / \mu \mathrm{s} \cdot \mu \mathrm{W}$ large-signal figure of merit (FOM) and $17 \mathrm{MHz} \cdot \mathrm{pF} / \mu \mathrm{W}$ smallsignal FOM with $1.2-\mathrm{V}$ supply voltage. In subthreshold, the op-amp has $10 \mathrm{~V} \cdot \mathrm{pF} / \mu \mathrm{s} \cdot \mu \mathrm{W}$ large-signal FOM and $92 \mathrm{MHz} \cdot \mathrm{pF} / \mu \mathrm{W}$ small-signal FOM with $0.5-\mathrm{V}$ supply voltage.
\end{abstract}

Index Terms-Analog integrated circuits, class AB-AB Miller op-amps, Miller compensation, resistive local common mode feedback.

\section{INTRODUCTION}

$\mathbf{T}$ HE increasing demand for battery-operated portable electronics equipment requires power-efficient analog circuits. The operational amplifier (op-amp) is the essential building block of analog signal processing units [1], [2]. Due to the reduction of the supply voltage in deep submicrometer mixed-signal design, the op-amp may suffer from slew rate limitation, insufficient bandwidth, lower gain, reduced dynamic range, inadequate noise performance, and linearity problems [3]. Hence, the energy-efficient high-speed

Manuscript received March 4, 2019; revised April 28, 2019; accepted May 12, 2019. Date of publication June 14, 2019; date of current version September 25, 2019. This work was supported by Grant TEC2016-80396C2-R (AEI/FEDER). (Corresponding author: Anindita Paul.)

A. Paul is with the Klipsch School of Electrical and Computer Engineering, New Mexico State University, Las Cruces, NM 88003 USA (e-mail: apau103@nmsu.edu; jairamir@nmsu.edu).

J. Ramírez-Angulo is with the Klipsch School of Electrical and Computer Engineering, New Mexico State University, Las Cruces, NM 88003 USA, and also with the National Institute of Astrophysics, Optics and Electronics, INAOE, Puebla 72000, Mexico.

A. J. López-Martín is with the Department of Electrical and Electronic Engineering, Public University of Navarra, E-31006 Pamplona, Spain (e-mail: antonio.lopez@unavarra.es).

R. G. Carvajal is with the Department of Electronic Engineering, School of Engineering, University of Seville, 41092 Seville, Spain (e-mail: carvajal@us.es).

J. M. Rocha-Pérez is with the National Institute of Astrophysics, Optics and Electronics, INAOE, Puebla 72000, Mexico (e-mail: jmigue11014@gmail.com).

Color versions of one or more of the figures in this article are available online at http://ieeexplore.iee.org.

Digital Object Identifier 10.1109/TVLSI.2019.2918235

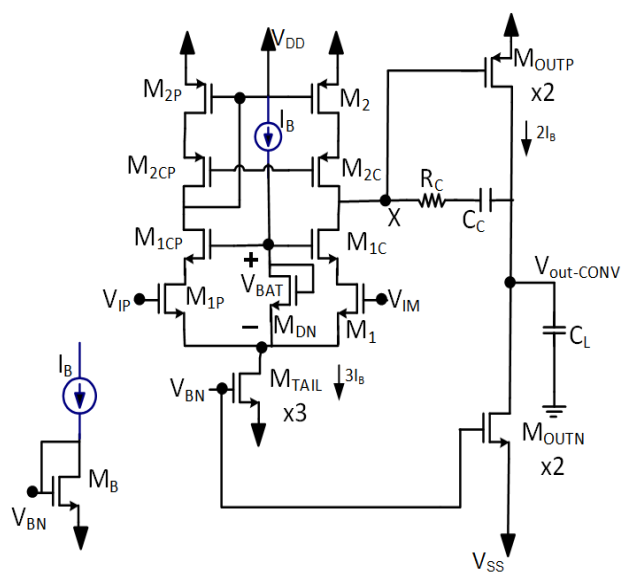

Fig. 1. Conventional high gain class-A two-stage Miller op-amp with telescopic input stage.

op-amp design is a challenging task for the analog designer in today's energy-constrained electronic world. Along with the low power and high speed, the op-amp must have a largesignal swing, high linearity, and high load driving capability. To achieve close to rail-to-rail output swing in low-voltage applications, a two-stage Miller op-amp is a wise choice. In this paper, a simple design approach of a Miller op-amp is presented to enhance gain-bandwidth product (GBW) by a large factor (63 times in the circuit presented here) and the $\mathrm{dc}$ open-loop gain by about one order of magnitude with just moderate increase ( $\sim$ factor 2$)$ in static power dissipation with respect to the conventional op-amp of Fig. 1. The approach also provides class $\mathrm{AB}$ operation to the input stage, improving the slew rate (SR). The efficiency of the op-amp is validated by measurements of a test chip and compared to other op-amps reported in the literature in terms of three figures of merit (FOMs): 1) the large-signal $\mathrm{FOM}_{\mathrm{LS}}=\left(\mathrm{SR} . C_{L}\right) / P_{Q}$, where $P_{Q}$ is the total static power dissipation and $C_{L}$ is the load capacitance; 2 ) the small-signal $\mathrm{FOM}_{\mathrm{SS}}=\left(\mathrm{GBW} \cdot C_{L}\right) / P_{Q}$; and 3) a global FOM $\mathrm{FOM}_{G}=$ $\left(\mathrm{FOM}_{L S} \mathrm{FOM}_{S S}\right)^{1 / 2}$. In order to account for silicon area and total quiescent current $\left(I_{\text {Total } Q}\right.$, four additional FOM are also reported here: $\mathrm{AFOMs}=\left(\mathrm{SR} . C_{L}\right) /\left(P_{Q}\right.$. Area $), \mathrm{AFOM}_{L}=$ $\left(\mathrm{GBW} . C_{L}\right) /\left(P_{Q} \cdot\right.$ Area $)$, IFOMs $=\left(\right.$ GBW. $\left.C_{L}\right) /\left(I_{\text {Total } Q}\right)[4]$, and $\mathrm{IFOM}_{L}=\left(\right.$ SR. $\left.C_{L}\right) /\left(I_{\text {Total } Q}\right)[4]$.

This paper is organized as follows. Section II summarizes the limitations of a conventional two-stage class-A Miller op-amp. Section III describes the proposed op-amp designed 
in 130-nm CMOS technology operating in strong inversion with $\pm 600-\mathrm{mV}$ dual supplies and a bias current $I_{B}=14 \mu \mathrm{A}$; and in subthreshold with \pm 250 -mV supply voltages and a bias current $I_{B}=70 \mathrm{nA}$. Section IV presents simulation results. Section V provides the experimental results of test chip prototypes of the proposed and conventional op-amps both in strong inversion and subthreshold. Conclusions are drawn in Section VI.

\section{TWO-StAGE Class-A Miller OP-AMP}

A two-stage Miller op-amp with a telescopic input stage is shown in Fig. 1. This circuit can provide high openloop dc gain, $A_{\mathrm{OLDC}} \sim\left(g_{m} r_{0}\right)^{3} / 4$, where $g_{m}$ and $r_{0}$ are transconductance gain and output resistance of a unit size transistor, respectively. For simplicity, the parameters $g_{m}$ and $r_{0}$ are considered equal here for all unit size transistors. It also provides a moderate gain bandwidth product (GBW).The GBW of the class A op-amp of Fig. 1 can be obtained from its dc open-loop gain and the dominant pole at node $X$ is given by the following equation:

$$
f_{\mathrm{PDOM}}=1 /\left(2 \pi R_{X} C_{X}\right) .
$$

Here, $R_{X}=g_{m} r_{0}^{2} / 2$ is the impedance at node $X$ and $C_{X}$ is the Miller capacitance given by the following equation:

$$
C_{X}=\left(1+A_{I I}\right) C_{C} .
$$

Here, $A_{I I}=g_{m} r_{0} / 2$ is the gain of the output stage. As the dc open-loop gain of the op-amp is $A_{\mathrm{OLDC}}=\left(g_{m} r_{0}\right)^{3} / 4$, the gain-bandwidth product is $\mathrm{GBW}_{\mathrm{cnv}}=g_{m} / 2 \pi C_{C}$. The high-frequency pole $f_{\text {Pout }}$ at the output node of the op-amp is given by the following equation:

$$
f_{\text {POut }}=g_{m \text { OUTP }} /\left(2 \pi C_{L}\right)
$$

Here, $g_{m \text { OUTP }}$ is the transconductance gain of the pMOS output transistor $\mathrm{M}_{\mathrm{OUTP}}$ and $C_{L}$ is the load capacitance. To achieve a high phase margin $\mathrm{PM}>50^{\circ}$ when the zero introduced by $C_{C}$ is shifted to infinity (by selecting $R_{C}=$ $\left.1 / g_{m \text { OUTP }}\right)$, it is common to select $C_{C}=C_{L}$ with transistors at the output stage scaled-up by a factor of 2 . In this case, the GBW has a value $\mathrm{GBW}_{\mathrm{cnv}}=g_{m} / 2 \pi C_{L}$ and the highfrequency pole has a value $f_{\text {Pout }}=2 \mathrm{GBW}_{\text {cnv }}$.

Both GBW and $f_{\text {POut }}$ can be increased by a factor $N$ by selecting $C_{C}=C_{L} / N$ and scaling-up the output transistors by a factor $2 N$. Unfortunately, this increases the bias current and the quiescent power dissipation of the op-amp of Fig. 1 by a factor $F=0.4(\mathrm{~N}+1.5)$ and maintains the open-loop gain constant.

A drawback of this circuit is the asymmetrical slew rate. Though it can have a large positive slew rate $\left(\mathrm{SR}^{+}\right)$, its negative slew rate $\left(\mathrm{SR}^{-}\right)$is constrained by the bias current of the output stage $I_{\text {OUTQ }}=2 I_{B}$. Hence, to improve $\mathrm{SR}^{-}$, it is required to increase $I_{\mathrm{OUTQ}}$ which increases the static power dissipation. Slew rate (SR) can be enhanced without increasing static power dissipation by utilization of a class $\mathrm{AB}$ (push-pull) output stage. The SR of the input stage (given by $\mathrm{SR}_{\text {inp }}=I_{\text {inp }} / C_{C}$, where $I_{\text {inp }}=2 I_{B}$ is the maximum current generated at node $X$ by the input stage) can also limit the SR

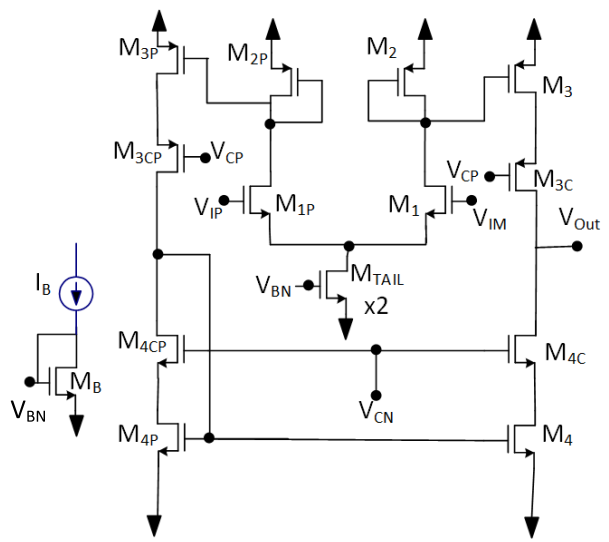

Fig. 2. Cascoded current mirror OTA [5].

of the op-amp since the input stage has to provide current to the compensation capacitor $C_{C}$ at node $X$. For this reason, it is convenient that the op-amp has also a class $\mathrm{AB}$ input stage that can generate $I_{\text {inp }}>2 I_{B}$.

Though class $A B$ operation can improve the current efficiency of the op-amp and decrease the linear part of the settling time, the exponential part of the settling time, determined by GBW, and phase margin (PM) can still lead to long total settling times in class $\mathrm{AB}$ op-amps. Hence to implement a high-speed op-amp both SR and GBW need to be improved simultaneously [5].

Power constraints in modern VLSI design demand an approach that can simultaneously increase GBW, SR, and open-loop gain. In Section III, an op-amp is proposed which can achieve large dc open-loop gain, enhanced GBW, and higher SR with just moderate increase in quiescent power. This op-amp can also work in subthreshold with little modification as shown in Section III-I. The small-signal and large-signal analysis for both op-amps are similar.

\section{Proposed CiRcuit}

\section{A. Operating Principle of the Proposed Op-Amp}

The proposed Class AB-AB Miller op-amp is shown in Fig. 3. It is a pseudo-three-stage op-amp derived from the conventional class-A op-amp of Fig. 1 by replacing the input stage with a modified version of the cascoded current mirror OTA [6] shown in Fig. 2 and a free class AB [7] output stage. The diode-connected transistors $M_{2}, M_{2 \mathrm{P}}$ of the current mirror OTA are replaced by a resistive local common mode feedback (RLCMFB) load [8]. The second stage $\left(M_{3}-M_{4}\right)$ is a high gain fully cascoded amplifier (shown in blue). The combination of first and second stages is denoted as "composite input stage" $\left(M_{1}-M_{4}\right)$. It is framed by a blue dotted line in Fig. 3. The resistors $R_{\mathrm{CM}}$ are selected so that the first stage $\left(M_{1}-M_{2}\right.$ shown in red and $R_{\mathrm{CM}}$ ) of the composite input stage provides moderate gain and generates high-frequency poles $\omega_{P X}$ at nodes $X$ and $X^{\prime}$ that have a negligible effect on the phase margin of the op-amp.

The output (third) stage ( $M_{\mathrm{OUTP}}$ and $M_{\mathrm{OUTN}}$ ) is a push-pull amplifier with the free class-AB operation. The RLCMFB with two matched resistors $\left(R_{\mathrm{CM}}\right)$ in conjunction with the scaling of 


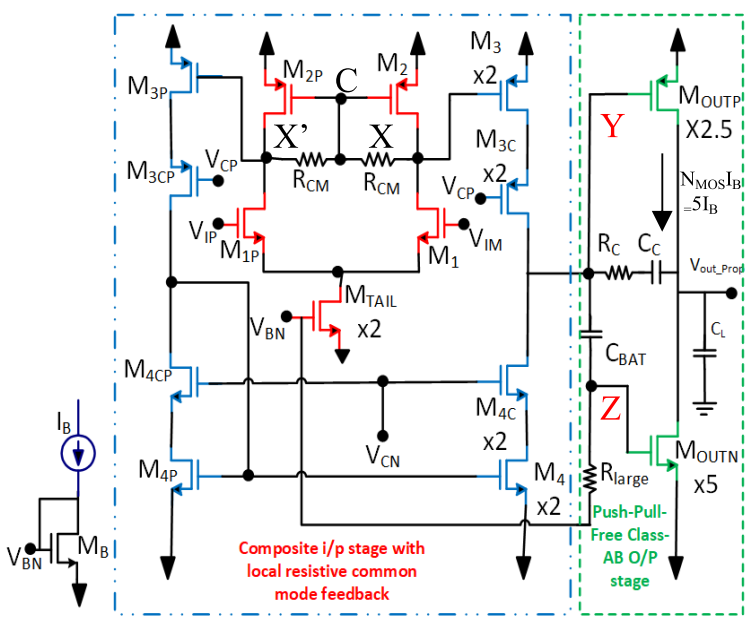

Fig. 3. Proposed class $\mathrm{AB}-\mathrm{AB}$ op-amp with $\mathrm{N}_{\mathrm{MOS}}=5$.

$M_{3}, M_{4}$ by a factor 2 provides: 1) approximately one order of magnitude enhanced effective transconductance $g_{m e f f}$, openloop gain, and higher GBW and 2) class $\mathrm{AB}$ operation at the op-amp's internal node Y. The output node of the composite input stage (node $\mathrm{Y}$ ) has high output resistance $R_{Y}$, and thus yields high gain. The output stage provides moderate gain, close to rail-to-rail output swing and class $\mathrm{AB}$ operation with comparably high positive and negative peak output currents. The class $\mathrm{AB}$ operation of both input and output stages of the op-amp prevents SR limitation of node $Y$ by the bias current of the first stage. It results in high SR, and hence high FOM $M_{\mathrm{LS}}$. The class $\mathrm{AB}$ output stage has the added advantage that it reduces the output impedance at high frequencies. This shifts the high-frequency output pole to higher frequencies, which improves the phase margin of the op-amp. The enhanced transconductance of the composite input stage $g_{m \text { eff }}$ leads to essential improvement in GBW and in the dc open-loop gain. This results in much higher $\mathrm{FO} M_{\mathrm{SS}}$ and high phase margin, with just moderate additional power consumption as discussed in the following analysis.

\section{B. Analysis of Class AB Operation of Input Stage}

In this section, it is shown that besides high gain, the input stage of the proposed op-amp has class $\mathrm{AB}$ operation and can deliver currents to $C_{C}$ at node $\mathrm{Y}$, which are larger than the static currents $\left(2 I_{B}\right)$ of transistors $M_{3}$ and $M_{4}$. Under quiescent conditions, no current flows through the RLCMFB resistors $R_{\mathrm{CM}}$. Hence, $M_{2}-M_{3}$ and $M_{2 \mathrm{P}-3 \mathrm{P}}$ behave as conventional current mirrors at dc. The branch with $\mathrm{M}_{3}$ in the second stage of the composite input stage is scaled up by a factor of 2 and has a quiescent current $2 I_{B}$. In the presence of a differential input voltage $V_{i d}$, a signal current $i=g_{m} V_{i d} / 2$ flows through the $R_{\mathrm{CM}}$ resistors and generates complementary voltage variations $\Delta V_{X}, \Delta V_{X}^{\prime}$ across each $R_{\mathrm{CM}}$. The effective resistance at nodes $X$ and $X^{\prime}$ is

$$
R_{X}=\left(R_{\mathrm{CM}}|| r_{o 1} \| r_{o 2}\right) .
$$

$R_{\mathrm{CM}}$ should be smaller than the output resistance of transistors $M_{1}$, 2, i.e., $R_{\mathrm{CM}}<<r_{\mathrm{O} 1,2}$ so that $R_{X} \approx R_{\mathrm{CM}}$. Complementary voltage variations with value $\Delta V_{X}=i R_{X}$ appear at nodes $X$, $X^{\prime}$ with maximum swing $\Delta V_{X \max }=I_{B} R_{X}$. The maximum source-gate voltage of transistors $M_{3}$ and $M_{3 \mathrm{P}}$ is

$V_{\mathrm{SG} 3 \max }=V_{\mathrm{SG} 3 \mathrm{Q}}+\Delta V_{X \max }=|V \mathrm{th}|+V$ SDsat $+I_{B} R_{X}$.

Transistor $M_{3}$ in the second stage can provide a maximum current $I_{3 \max }$ at node $Y$ which is given by the following equation:

$$
\begin{aligned}
I_{3 \max } & =\beta_{3}\left(V_{\mathrm{SG} \max 3}-\left|V_{\mathrm{th}}\right|\right)^{2} \\
& =\left.\beta 3\left(V_{\mathrm{SD} \mathrm{sat} 3}+\left|V_{\mathrm{th}}\right|+I_{B} R_{X}-\mid V_{\mathrm{th}}\right)\right|^{2} \\
\beta_{3} & =\frac{1}{2} \mu_{P} C_{\mathrm{OX}}\left(\frac{W}{L}\right)_{3} .
\end{aligned}
$$

Defining $M=\left(I_{B} R_{X}\right) / V_{\text {SDsat } 3}, I_{3 \max }$ can be expressed by the following equation:

$$
I_{3 \max }=\beta_{3}(M+1)^{2} V_{\mathrm{SD} \text { sat } 3}^{2}=I_{B 3 Q}(M+1)^{2} .
$$

Hence, depending on the value of $R_{X}$ and $I_{B}$, resistors $R_{\mathrm{CM}}$ can help to increase the positive and negative peak currents $I_{\text {peakY }}$ delivered to the compensation capacitor $C_{C}$ at node $Y$. Design values of $R_{\mathrm{CM}} \approx R_{X}=16 \mathrm{k} \Omega,\left|V_{\text {DSsat } 3}\right|=160 \mathrm{mV}$, $I_{B}=14 \mu \mathrm{A}$, and $I_{B 3 Q}=2 I_{B}=28 \mu \mathrm{A}$ were selected here. These selections result in $M+1 \approx 2.4$, which leads to a current $I_{3 \max }=6 I_{B 3 Q}=12 I_{B}=168 \mu \mathrm{A}$ at node Y. Increasing $R_{X}$ results in higher $M$ values (and higher gain), but this decreases the high-frequency poles at nodes $X, X^{\prime}$, and can lead to a reduction of the phase margin of the op-amp, as discussed in Section III-E.

\section{Class AB Operation of Output Stage}

To improve the SR, it is also necessary to increase the negative peak current of the output stage. This is done based on the free class $\mathrm{AB}$ technique [7]-[9] by means of a large resistance $R_{\text {Large }}$ and as mall capacitor $C_{\mathrm{BAT}}$ that provides dynamic class AB operation at the output node of the op-amp. Under quiescent conditions, $R_{\text {Large }}$ provides equal bias voltage $V_{\mathrm{BN}}$ to $M_{\mathrm{TAIL}}$ and $M_{\mathrm{OUTN}}$, and consequently currents $2 I_{B}$ and $N_{\text {MOS }} I_{B}$ flow through $M_{\text {TAIL }}$ and the two output transistors $M_{\text {OUTN }}$ and $M_{\text {OUTP }}$, respectively. $M_{\text {OUTN }}$ is scaled by a factor $N_{\mathrm{nMOS}}=2 N=5$ in the proposed design. This leads to a quiescent current $I_{\text {out } Q}=5 I_{B}$ in the output branch transistors. Under dynamic conditions, when the op-amp is slewing in the negative direction, the voltage at node $Y$ faces a positive change. Due to the presence of the large valued resistor, the capacitor cannot charge or discharge rapidly. Hence, it acts as a floating battery and transfers the voltage variations from node $Y$ to node $Z$. Hence, a large dynamic negative current (higher than the quiescent output current $N_{\mathrm{MOS}} I_{B}$ ), can be obtained at the op-amp's output node, improving current efficiency $\mathrm{CE}=I_{\mathrm{O} \text { max }} / I_{\mathrm{Total} Q}$ where $I_{\mathrm{O} \text { ax }}$ is the maximum output current and $I_{\text {Total } Q}$ is the total op-amp's quiescent current.

\section{Open-Loop-Gain Analysis of the Proposed Op-Amp}

The simplified small-signal model of the proposed op-amp is shown in Fig. 4, leading to three poles: two at the internal 
This is the author's version of an article that has been published in this journal. Changes were made to this version by the publisher prior to publication. The final version of record is available at http://dx.doi.org/10.1109/10.1109/TVLSI.2019.2918235

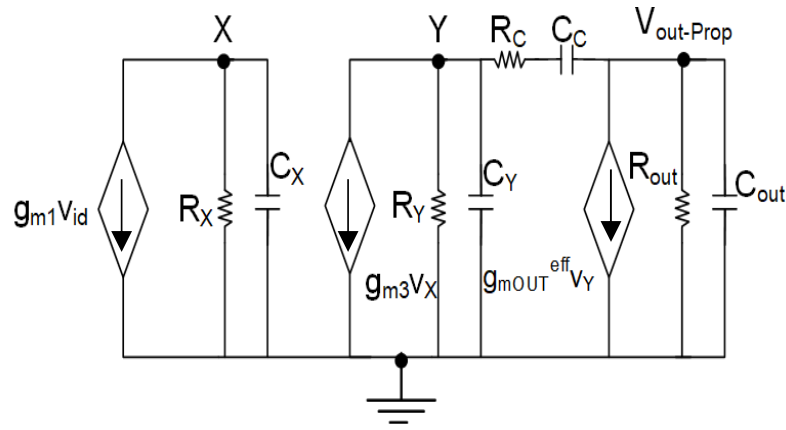

Fig. 4. Small-signal model of the proposed class AB-AB op-amp.

nodes $X$ and $Y$, and one at the output node. The feedforward path formed by $R_{C}$ and $C_{C}$ creates a zero. The corresponding open-loop transfer function $A_{\mathrm{OL}}(s)$ of the proposed op-amp is expressed by the following equation:

$$
A_{\mathrm{OL}}(s)=\frac{A_{I} A_{I I} A_{I I I}\left(1+s / \omega_{z}\right)}{\left(1+s / \omega_{\mathrm{PDOM}}\right)\left(1+s / \omega_{\mathrm{POut}}\right)\left(1+s / \omega_{\mathrm{PX}}\right)}
$$

where $A_{I}, A_{I I}$, and $A_{I I I}$ are the dc gains of the first, second, and third stage, respectively. Gain $A_{I}$ is $A_{I}=\left(V_{X}-\right.$ $\left.-V_{X^{\prime}}\right) / V_{i d}=g_{m 1} R_{X}$. Cascoded transistors $M_{3 \mathrm{C}}$ and $M_{4 \mathrm{C}}$ are used to increase the gain of the second stage $A_{I I}=g_{m 3} R_{Y}$. $R_{Y}$ is expressed by the following equation assuming that all $r_{o}$ 's are equal and $g_{m 3}=g_{m 4}=g_{m 3 C}=g_{m 4 C}$ :

$$
R_{Y}=\left(g_{m 3 C} r_{o 3 C} r_{o 3}\right) \|\left(g_{m 4 C} r_{o 4 C} r_{o 4}\right) \approx g_{m} r_{o 3}^{2} / 2 \text {. }
$$

The resultant dc open-loop gain from the composite input stage is

$$
A_{\text {input }}=A_{I} A_{I I}=g_{m 1} R_{X}\left(g_{m 3} r_{o 3}\right)^{2} / 2 .
$$

The output stage consists of a push-pull amplifier that provides moderate gain and close to rail-to-rail output swing. The dc gain of the output stage is $A_{I I I}=\left(g_{m \text { OUTP }}\right)\left(r_{\text {OUTP }} \| r_{\text {OUTN }}\right)$. Hence, the total dc open-loop gain is given by AOLDC

$$
\begin{aligned}
A_{\mathrm{OLDC}} & =A_{I} A_{I I} A_{I I I} \\
& =\left(g_{m 1} R_{X}\right) \frac{\left(g_{m 3} r_{o 3}\right)^{2}}{2} g_{\mathrm{mOUTP}}\left(r_{\mathrm{OUTP}} \| r_{\mathrm{OUTN}}\right) \\
A_{\mathrm{OLDC}} & \approx g_{m} R_{\mathrm{CM}}\left(g_{m} r_{o}\right)^{3} / 4 .
\end{aligned}
$$

\section{E. Pole-Zero Analysis of the Proposed Op-Amp}

Equation (8) shows that the op-amp has three poles and one zero. The selection of $R_{\mathrm{CM}}$ plays an important role in this design, as it poses a tradeoff between the boosting of the gain $A_{I}=g_{m 1} R_{X}$ in the input stage, the value of the highfrequency poles $\left(\omega_{P X}\right)$ at node $X\left(X^{\prime}\right)$, and the peak current $I_{3 \max }$ at node $Y$, as discussed in Section III-B. The pole of the circuit at node $X, X^{\prime}$ is given by the following equation:

$$
\omega_{P X}=1 / R_{X} C_{X} \approx 1 / R_{C M} C_{X} .
$$

Node $C$ at the input stage operates with constant voltage (ac ground). Due to this, the effects of $C_{\mathrm{GS} 2,2 \mathrm{P}}$ are nullified at node $X$. Thus, the parasitic capacitance at node $X$ is given by the following equation:

$$
C_{X}=C_{\mathrm{GS} 3}+C_{\mathrm{db} 2}+C_{\mathrm{db} 1}+C_{\mathrm{GD} 1}+C_{\mathrm{GD} 2} .
$$

Since $C_{X}$ is very small and $R_{X} \ll r_{o}, \omega_{P X}$ is a high-frequency pole (much higher than GBW), as shown later in Section III-G. In this case, its effect can be neglected, and $A_{\mathrm{OL}}(s)$ of the opamp can be approximated by a conventional two-pole one-zero system and given by the following equation:

$$
A_{\mathrm{OL}}(s) \approx \frac{A_{I} A_{I I} A_{I I I}\left(1+s / \omega_{z}\right)}{\left(1+s / \omega_{\mathrm{PDOM}}\right)\left(1+s / \omega_{\mathrm{POut}}\right)} .
$$

The dominant pole at node $Y$ is given approximately by the following equation:

$$
\omega_{\mathrm{PDOM}} \approx \frac{1}{R_{Y} A_{I I I} C_{c}} .
$$

The GBW (in hertz) of the op-amp in Fig. 3 is given in the following equation:

$$
\begin{aligned}
\mathrm{GBW} & =A_{\mathrm{OLDC}} \omega_{\mathrm{PDOM}}=A_{I} A_{I I} A_{I I I} \omega_{\mathrm{PDOM}} \\
& \approx\left(A_{I}\left(g_{m 3} R_{Y}\right) A_{I I I}\right) /\left(R_{Y} C_{C} A_{I I I}\right) \\
& \approx A_{I} g_{m 3} / C_{C}=g_{m \mathrm{eff}} /\left(2 \pi C_{C}\right) .
\end{aligned}
$$

Equation (16) implies that the effective transconductance of the composite input stage and of the op-amp of Fig. 3 is given by $g_{m e f f}=A_{I} g_{m 3}$. Hence, $A_{I}$, in conjunction with the factor 2 scaling of $M_{3}$, increases the effective transconductance, the GBW, and the dc open-loop gain of the op-amp approximately by a factor $2 A_{I}$ at the expense of a moderate increase in power dissipation and the introduction of the highfrequency poles $\omega_{P X}$. In the proposed design, the value of $R_{\mathrm{CM}}$ and the scaling by a factor 2 of $M_{3}$ in the second stage provides a gain boosting in the composite input stage by a factor close to 10 .

The nondominant pole in (14) corresponds to the output terminal and is given by the following equation:

$$
\omega_{\mathrm{POut}}=\left(g_{m \mathrm{OUT}}^{\mathrm{eff}} / C_{L}\right) \text {. }
$$

Here, $g_{m \text { OUT }}^{\text {eff }}=g_{m \text { OUTP }}+g_{m \text { OUTN }}$ is the output conductance of the op-amp at high frequencies. The zero is given by the following equation:

$$
\omega_{z}=\frac{1}{\left(C_{C} R_{C}^{\mathrm{eff}}\right)}
$$

where $R_{C}^{\text {eff }}=\left(R_{C}-1 / g_{m \text { OUTT }}{ }^{\text {eff }}\right)$.

Now, to achieve high GBW and high phase margin with low silicon area, $C_{C}$ is selected to have a moderate-low value $C_{C}=C_{L} / 10$ and $R_{C}$ is selected so that the output pole $\omega_{P \text { Out }}$ matches the zero $\omega_{z}$. In this case, $\omega_{P \text { out }}$ and $\omega_{\mathrm{z}}$ can be lower than GBW, but the op-amp can still have high phase margin. This is discussed in detail in Section III-F with a design example.

The proposed approach enhances the open-loop gain and the effective $g_{m e f f}$ of the op-amp by providing an additional gain in the first stage with a negligible impact on the PM. This is possible in current technology (like in $0.13-\mu \mathrm{m}$ CMOS technology and finer technologies) since the value of $C_{X}$ is 
very small. Thus, $\omega_{P X} \gg$ GBW. Hence, the enhancement of $g_{m \text { eff }}$ improves GBW and the dc open-loop gain. On the contrary, using RLCMFB to enhance gain in previous technologies (like in $0.5-\mu \mathrm{m}$ technology) [8], the value of $C_{X}$ was not so small and the poles at node $X$ and $X^{\prime}$ could be lower or close to GBW. So, in order to achieve a high PM phase, lead compensation was required. This uses a left half $s$ plane zero generated by a resistor $R_{\mathrm{S}}$ connected in series with $C_{L}$ to compensate for the phase shift of $\omega_{P X}$ which can limit the op-amp's maximum dynamic output current. Thus, this proposed op-amp in 130-nm technology does not require phase lead compensation if the RLCMFB provides only moderate gain enhancement in the input stage.

\section{F. Stability Analysis}

As mentioned above [see (18)], in general for stability with $\mathrm{PM}>50^{\circ}$, the Miller op-amp is designed with $R_{C}=1 / g_{m \text { OUT }}^{\text {eff }}$ to shift the right half- $s$ plane zero to infinity and the highfrequency output pole $\omega_{P \text { Out }}$ must be higher than GBW by at least a factor of 2. As indicated in Section II, a common practice to achieve this is to scale the output transistors by a factor 2 and to select $C_{C}=C_{L}$. In this case, the highfrequency output pole limits the maximum value of GBW to a value $f_{P \text { Out }} / 2=g_{m} /\left(2 \pi C_{L}\right)=g_{m} /\left(2 \pi C_{C}\right)$. As discussed in Section II, GBW can be increased by a factor $N$ by selecting $C_{C}=C_{L} / N$ and scaling the output transistors by a factor of $2 N$. This increases quiescent power dissipation by a factor of $0.4(N+1.5)$ and maintains the same dc open-loop gain.

The approach followed here allows to achieve higher GBW values with lower power dissipation as shown below. It consists of matching the value of the zero $\omega_{z}$ to the output pole $\omega_{\text {POut }}$. From (17) and (18), the condition for pole-zero matching $\omega_{\mathrm{POut}}=\omega_{z}$ leads to $R_{C}=\left(1+C_{L} / C_{C}\right) / g_{m \text { OUT }}^{\text {eff }}$

Consider, for example, the design of the proposed circuit of Fig. 3 with scaling factors $N_{\mathrm{pMOS}}=2.5$ and $N_{\mathrm{nMOS}}=$ $5\left(I_{\text {OUTQ }}=5 I_{B}\right)$ in the output stage transistors $M_{\text {OUTP }}$ and $M_{\text {OUTN }}$, respectively, with $C_{C}=C_{L} / 10, R_{\mathrm{CM}}=16 \mathrm{k} \Omega$, and a factor 2 scaling of transistor $M_{3}$. The free class $\mathrm{AB}$ output stage can provide a $g_{m \text { OUT }}^{\text {eff }}$ value given by the following equation:

$$
g_{m \mathrm{OUT}}^{\text {eff }}=\left(g_{m \text { OUTP }}+g_{m \text { OUTN }}\right) \text {. }
$$

As $g_{m, n}=\left(2 I_{B} \mu_{n, p} C_{\mathrm{ox}}(W / L)_{n, p}\right)^{1 / 2}, \quad g_{\text {mOUT }}^{\text {eff }}=$ $\left(\left(N_{\mathrm{nMOS}} N_{\mathrm{pMOS}}+N_{\mathrm{nMOS}}\right)^{1 / 2}\right) g_{m 1}=8.5 \mathrm{gm} 1$. The scaling of the output transistors shifts both the high-frequency output pole (and the matching zero) by almost an order of magnitude. Hence, this approach, in conjunction with the enhancement of $g_{m \mathrm{OUT}}^{\text {eff }}$ and the reduction of $C_{C}$ leads to an essentially higher GBW value than the conventional op-amp and places the output pole and zero at higher values, such that high PM is possible. Note that the enhancement of GBW is also possible due to the enhancement of $g_{m e f f}$ introduced by the RLCMFB and by the factor of 2 scaled-up transistors $M_{3}$ and $M_{3 \mathrm{P}}$ in the composite input stage. In the proposed approach, a value $\mathrm{GBW}=A_{I} g_{m 3} / C_{C}$ is obtained from (16) where $A_{I} \approx 3.5$ is the gain introduced by the RLCMFB in the first stage. Thus, $g_{\text {meff }}$ is enhanced by a factor of $2 A_{I} \approx 7$.

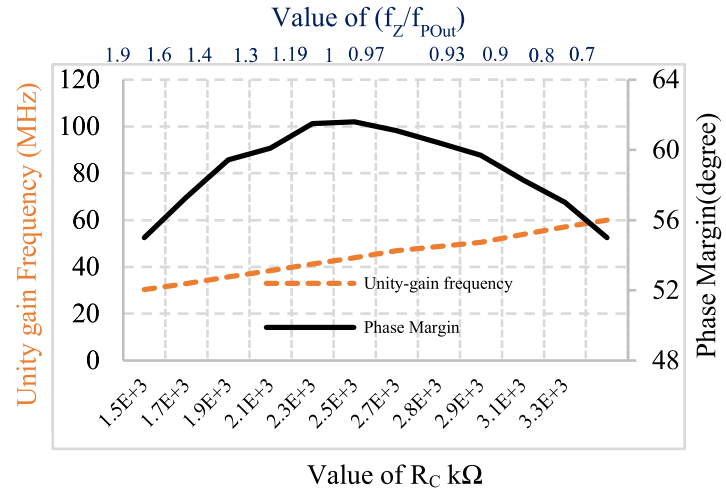

Fig. 5. Variation of phase margin, unity-gain frequency, and pole-zero mismatch with $\mathrm{R}_{\mathrm{C}}$.

Scaling down $C_{C}$ by a factor 10 provides additional GBW enhancement by the same factor. Hence GBW of the proposed circuit is given by $\mathrm{GBW}_{\text {prop }}=2 A_{I}\left(g_{m} / 2 \pi C_{c}\right)=20 A_{I}$ $\left(g_{m} / 2 \pi C_{L}\right)=70 \mathrm{GBW}_{\mathrm{cnv}}$. This is a factor 70 times higher than the GBW of the conventional approach. This is achieved by: 1) enhancing $g_{m e f f}$ in three steps in the input stage; 2) scaling down $C_{C}$; and 3 ) matching the high-frequency pole to the high-frequency zero. Downscaling of capacitor $C_{C}$ also saves silicon area and simultaneously relaxes the SR requirements at the internal node $Y$ of the composite input stage. Enhancing $g_{m e f f}$ also enhances the dc open-loop gain by a factor $2 A_{I}$.

Fig. 5 shows the variation of the PM and pole-zero mismatch factor $f_{z} / f_{\text {Pout }}$ as a function of $R_{C}$ obtained from simulations with $C_{L}=50 \mathrm{pF}$. According to (18), the changes in $R_{C}$ lead to changes in $\omega_{z}$. It is desirable to have a high PM. From Fig. 5, it can be observed that the approach proposed here is robust against $R_{C}$ manufacturing variations since PM $>60^{\circ}$ for $2 \mathrm{k} \Omega<R_{C}<2.8 \mathrm{k} \Omega$, which corresponds to a polezero mismatch range $0.93<f_{Z} / f_{\text {Pout }}<1$.4. PM also remains higher than $55^{\circ}$ in the range from $1.5 \mathrm{k} \Omega<R_{C}<3.3 \mathrm{k} \Omega$ and $0.8<f_{Z} / f_{\text {Pout }}<1.9$. It is noticeable that the phase margin is within $55^{\circ}$ for a large pole and zero mismatch factor between 0.7 and 1.9. Note that the accurate pole-zero matching is not required to achieve a high PM. However, pole-zero pairs (doublets) can affect the settling time $\left(t_{\text {Setl }}\right)$ of class $\mathrm{A}$ and class AB op-amps [10]. According to [11], the exponential part of the transient response of the op-amp to a step input of amplitude $\mathrm{V}$ is given by the following equation:

$V_{\text {Out }}(t)=V\left[1-\exp (-\mathrm{GBW} * t)+\frac{\omega_{z}-\omega_{p}}{\mathrm{GBW}} \exp \left(-\omega_{z} t\right)\right]$.

Here, $\omega_{z}$ and $\omega_{p}$ are pole and zero doublet frequencies. The third term can lead to long settling times if there is large relative pole-zero mismatch $\left(\omega_{z}-\omega_{p}\right) / \mathrm{GBW}$ and/or low pole-zero doublet frequencies relative to GBW: $\omega_{z}, \omega_{p} \ll \mathrm{GBW}$. In the design proposed here, $\omega_{z}$ and $\omega_{\mathrm{pOut}}$ are approximately a factor 4-5 lower than GBW. Simulations shown in Section IV-A with relatively large pole-zero mismatch values $\left(\omega_{z} / \omega_{\mathrm{pOut}}\right.$ in the range from 0.8 to 1.7) caused by variations in $R_{C}$ and $C_{L}$ show that even in these large ranges the pole-zero mismatches do not lead to long settling times in the proposed design. Fig. 6 


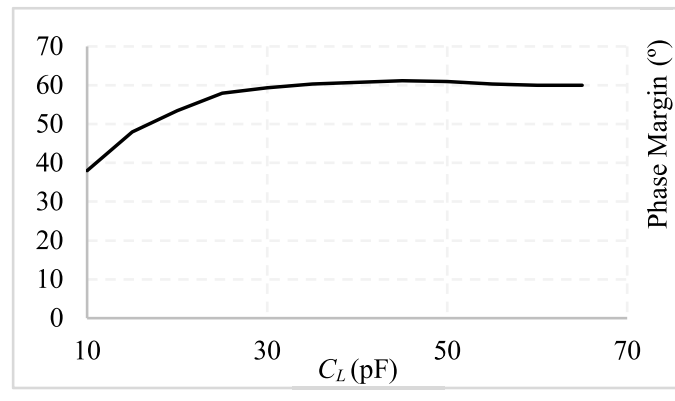

Fig. 6. Phase margin as a function of $\mathrm{C}_{L}$.

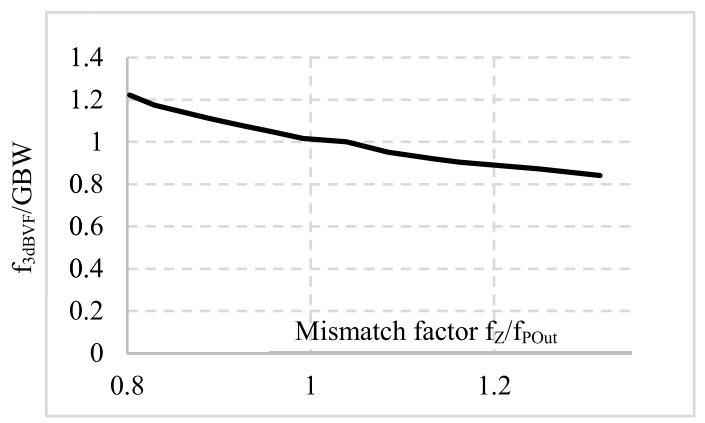

Fig. 7. $\mathrm{f}_{3 \mathrm{dBVF}} / \mathrm{GBW}$ as a function of $\mathrm{f}_{Z} / \mathrm{f}_{\text {Pout }}$.

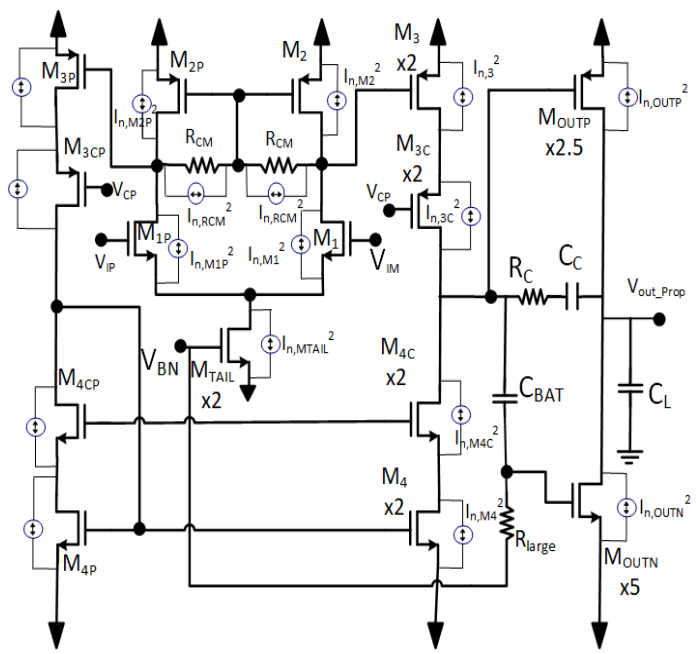

Fig. 8. Noise model of the proposed op-amp.

shows the phase margin of the op-amp as a function of $C_{L}$. It can drive a load $C_{L}=20 \mathrm{pF}$ with $\mathrm{PM}=54^{\circ}$.

The transfer function of the proposed op-amp in voltage follower (VF) configuration is given by the following equation:

$$
G_{\mathrm{CL}}(s)=\frac{A_{\mathrm{OLDC}}\left(1+\frac{s}{\omega_{z}}\right)}{\left(1+\frac{s}{\omega_{\mathrm{PDOM}}}\right)\left(1+\frac{s}{\omega_{\text {POut }}}\right)+A_{\mathrm{OLDC}}\left(1+\frac{s}{\omega_{z}}\right)} .
$$

For $f_{Z} / f_{\text {POut }}=1$ (pole matches the zero), (21) reduces to a one pole transfer function. In that case, the BW of the VF is $f_{3 \mathrm{dBVF}}=$ GBW. Fig. 7 shows $f_{3 \mathrm{dBVF}} / \mathrm{GBW}$ as a function of mismatch $f_{Z} / f_{\text {Pout }}$. It can be noticed that for the range of $0.8<f_{Z} / f_{\text {Pout }}<1.32, f_{3 \mathrm{dBVF} / \mathrm{GBW}}$ lies in the range of

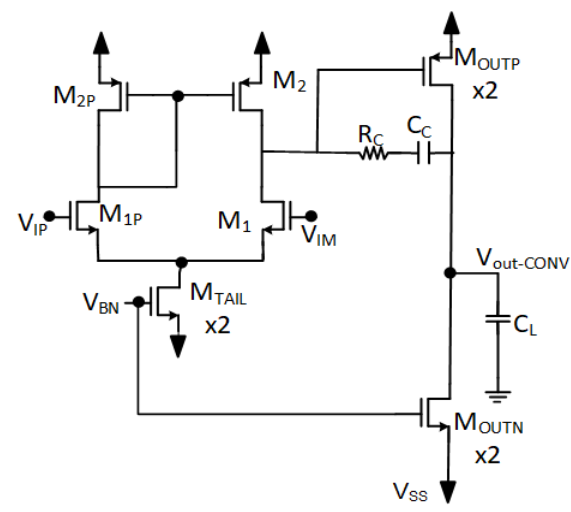

**(a)

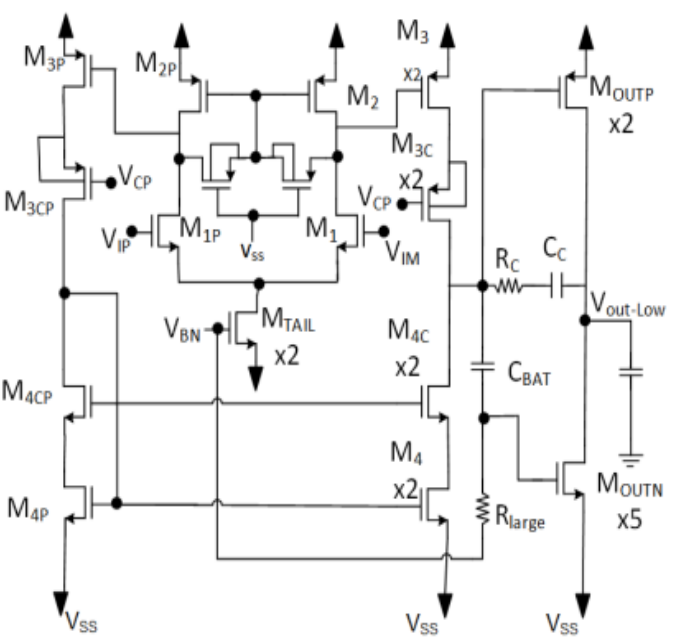

(b)

Fig. 9. (a) Subthreshold implementations of Conv-A op-amp. (b) Subthreshold implementations of the Proposed-AB-AB Op-amp. ${ }^{* *}$ No telescopic Conventional op-amp is considered here. Due to the low supply voltage constrain $( \pm 0.25 \mathrm{~V})$.

$0.8<f_{3 \mathrm{dBVF} / \mathrm{GBW}}<1.22$. Therefore, $f_{3 \mathrm{dBVF}}$ is not highly sensitive to pole-zero mismatch.

\section{G. Design Considerations}

Table I shows the design parameters of the proposed design (column 2) and of the conventional op-amp of Fig. 1 for two cases: 1) with $C_{C}=C_{L}$ and $R_{C}$ selected in such a way that $f_{z}$ is shifted to infinity (column 3) and 2) with $C_{C}=C_{L} / 10$ and $R_{C}$ selected to achieve pole-zero cancellation (column 4). In the proposed design the nominal value of the zero $f_{Z}$ is $16 \mathrm{MHz}$ obtained from (18) and the first high-frequency pole $f_{\text {POut }}$ is close to $14 \mathrm{MHz}$ from (17). Hence, the effect of the 1 st nondominant pole is approximately nullified by the lefthand plane zero. The dominant pole obtained from (15) has a value $f_{\mathrm{PDOM}}=4.8 \mathrm{kHz}$. The dc open-loop gain is $A_{\mathrm{OLDC}}=$ $82.3 \mathrm{~dB}$ from (11), and GBW $=63 \mathrm{MHz}$ from (16). From (12), the second-high-frequency pole is at $f_{p X}=200 \mathrm{MHz}$. Hence in this design, $f_{3 \mathrm{~dB}}$ in $\mathrm{VF}$ configuration is $57 \mathrm{MHz}$ for the values of $f_{Z}$ and $f_{\text {POut }}$. Notice that the conventional op-amp has a value $\mathrm{GBW}=12 \mathrm{MHz}$ with pole-zero cancellation and $C_{c}=C_{L} / 10$, while it has a value GBW $=1.1 \mathrm{MHz}$ with 
This is the author's version of an article that has been published in this journal. Changes were made to this version by the publisher prior to publication. The final version of record is available at http://dx.doi.org/10.1109/10.1109/TVLSI.2019.2918235

TABLE I

VALUes of Design Parameters of the Proposed AND CONV OP-AMP IN STRONG INVERSION

\begin{tabular}{|c|c|c|c|}
\hline Parameter & $\begin{array}{l}\mathrm{C}_{\mathrm{C}}=\mathrm{C}_{\mathrm{L}} / \mathbf{1 0} \\
\text { (proposed) }\end{array}$ & $\begin{array}{l}C_{C}=C_{L} \\
\text { Conv }\end{array}$ & $\begin{array}{l}\mathrm{C}_{\mathrm{C}}=\mathrm{C}_{\mathrm{L}} / 10 \\
\text { Conv }\end{array}$ \\
\hline$I_{B}(\mu \mathrm{A})$ & 14 & 14 & 14 \\
\hline$R_{\text {large }}(\mathrm{k} \Omega)$ & 500 & NA & NA \\
\hline$R_{C M}(\mathrm{k} \Omega)$ & 16 & NA & NA \\
\hline$C_{C}(\mathrm{pf})$ & 4.6 & 50 & 4.6 \\
\hline$C_{L}(\mathrm{pF})$ & 50 & 50 & 50 \\
\hline$R_{C}(\mathrm{k} \Omega)$ & 2.3 & 1 & 12 \\
\hline$C_{b a t}(\mathrm{pF})$ & 2 & NA & NA \\
\hline $\begin{array}{l}\text { NMOS(unit } \\
\text { transistor's } \\
\mathrm{W} / \mathrm{L})(\mu \mathrm{m})\end{array}$ & $5 / 0.18$ & $5 / 0.18$ & $5 / 0.18$ \\
\hline $\begin{array}{l}\text { PMOS(unit } \\
\text { transistor's } \\
\text { W/L) }(\mu \mathrm{m})\end{array}$ & $20 / 0.18$ & $20 / 0.18$ & $20 / 0.18$ \\
\hline $\begin{array}{l}g_{m l}, g_{m 3}, g_{m O U T P}, g_{m O} \\
U T N(\mu \mathrm{A} / \mathrm{V})\end{array}$ & $\begin{array}{l}311,492,1770 \\
2700\end{array}$ & $\begin{array}{l}351, N A, \\
931,1082\end{array}$ & $\begin{array}{l}351, \mathrm{NA}, \\
931,1082\end{array}$ \\
\hline $\begin{array}{l}g_{d s I}, g_{d s 2}, g_{d s 3}, \\
g_{d s O U T P,}, g_{d s O U T N} \\
(\mu \mathrm{A} / \mathrm{V})\end{array}$ & $\begin{array}{l}11.81,9.62 \\
21.33,50.54, \\
73.47\end{array}$ & $17,12, \mathrm{NA}, 26,33$ & $\begin{array}{l}\text { 17,12,NA,26, } \\
33\end{array}$ \\
\hline $\begin{array}{l}A_{O L D C}, \mathrm{GBW}, \\
f_{P D O M}, f_{p X}, f_{p O u t}, f_{z,} \\
t_{\text {Set }}{ }^{+}, t_{\text {Set }}, P M\end{array}$ & $\begin{array}{l}82.3 \mathrm{~dB}, \\
63 \mathrm{MHz}, \\
4.8 \mathrm{kHz}, 200 \mathrm{MHz}, \\
14 \mathrm{MHz}, 16 \mathrm{MHz}, \\
45 \mathrm{~ns}, 50 \mathrm{~ns}, 60^{\circ}\end{array}$ & $\begin{array}{l}70.5 \mathrm{~dB}, \\
1.1 \mathrm{MHz}, 311 \mathrm{~Hz} \text {, } \\
\mathrm{NA}, \quad 3 \mathrm{MHz} \text {, } \\
\text { infinity, } 1.2 \mu \mathrm{s}, \\
2 \mu \mathrm{s}, 70^{0}\end{array}$ & $\begin{array}{l}70.5 \mathrm{~dB}, \\
12 \mathrm{MHz}, \\
3.3 \mathrm{kHz}, \mathrm{NA}, \\
3 \mathrm{MHz}, \\
2.9 \mathrm{MHz}, \\
285 \mathrm{~ns}, 890 \mathrm{~ns} \\
98^{0}\end{array}$ \\
\hline
\end{tabular}

$C_{c}=C_{L}$ and $f_{z}$ shifted to infinity. In both cases, the GBWs are lower since the proposed $g_{m e f f}$ boosting technique is not used. The $0.1 \%$ positive and negative settling times $\left(t_{\text {Setl }}^{+}\right.$and $\left.t_{\text {Set } 1}^{-}\right)$of the proposed op-amp for the design parameters given in Table I are 45 and $50 \mathrm{~ns}$, respectively, whereas for the ConvA op-amp with $C_{C}=C_{L} / 10, t_{\text {Setl }}^{+}$and $t_{\text {Setl }}^{-}$are 285 and $890 \mathrm{~ns}$, respectively. For $C_{C}=C_{L}, t_{\text {Setl }}^{+}$and $t_{\text {Setl }}^{-}$are 1.2 and $2 \mu \mathrm{s}$, respectively.

\section{H. Noise Analysis}

The most significant sources of the internal noise in the opamp are the thermal noise of the MOSFETs and resistors and the flicker noise of the MOSFETs. A simple expression of the noise current spectral density of a MOSFET is [12]

$$
I_{n}^{2}(f)=4 \gamma k_{B} T g_{m}+\frac{K_{f} g_{m}^{2}}{\mathrm{WLC}_{\mathrm{ox}} f}=4 \gamma k_{B} T g_{m}+\frac{2 \mu K_{f} I_{D}}{L^{2} f}
$$

with $T$ the absolute temperature, $k_{B}$ the Boltzmann's constant, $\mu$ the carrier mobility, $K_{f}$ the flicker noise coefficient, and $\gamma$ a factor that varies from $1 / 2$ to $2 / 3$ from weak to strong inversion. The first term in (22) corresponds to thermal noise and the second term to flicker noise. The thermal noise of the resistor $R_{\mathrm{CM}}$ can also be modeled by a current source with a power spectral density of $I_{\mathrm{nRCM}}^{2}(f)=4 k_{B} T / R_{\mathrm{CM}}$. Fig. 8 shows the noise sources. Assuming that they are uncorrelated and that all transistors have the same $\gamma$ factor, the inputreferred mean-square thermal noise of the amplifier in a bandwidth $\Delta f$ is

$$
\begin{aligned}
& \overline{v_{\mathrm{nt}, \mathrm{in}}^{2}}=\frac{4 \gamma k_{B} T \Delta f}{g_{m 1}} \\
& \times\left[\begin{array}{c}
1+\frac{g_{m 1 P}}{g_{m 1}}+\frac{g_{m 2}}{g_{m 1}}+\frac{g_{m 2 P}}{g_{m 1}}+\frac{2}{\gamma g_{m 1} R_{\mathrm{CM}}}+1 /\left(g_{m 3} R_{\mathrm{CM}}\right)^{2} \\
\left(\frac{g_{m 3}}{g_{m 1}}+\frac{g_{m 4}}{g_{m 1}}\right)+1 /\left(g_{m 3 P} R_{\mathrm{CM}}\right)^{2}\left(\frac{g_{m 3 P}}{g_{m 1}}+\frac{g_{m 4 P}}{g_{m 1}}\right)
\end{array}\right] .
\end{aligned}
$$

The noise contribution of the output stage is not considered as it is divided by the high-gain of the input-stage when referred to the input. Noting that $g_{m 3}=g_{m 4}=2 g_{m 2}$ and $g_{m 3 P}=$ $g_{m 4 P}=g_{m 2 P}=g_{m 2}$ and $g_{m 1}=g_{m 1 P}$, from (23)

$$
\overline{v_{\mathrm{nt}, \mathrm{in}}^{2}}=\frac{8 \gamma k_{B} T \Delta f}{g_{m 1}}\left[\begin{array}{c}
1+\frac{g_{m 2}}{g_{m 1}}+\frac{1}{\gamma g_{m 1} R_{\mathrm{CM}}}+\frac{1}{g_{m 1} g_{m 3} R_{\mathrm{CM}}^{2}} \\
+\frac{1}{g_{m 1} g_{m 3 P} R_{\mathrm{CM}}^{2}}
\end{array}\right] .
$$

Note that for moderately large $g_{m 3} R_{\mathrm{CM}}$ and $g_{m 3 \mathrm{P}} R_{\mathrm{CM}}$ noise is dominated by the first stage.

Regarding flicker noise, assuming as before uncorrelated noise sources and the same $K_{f}$ for all the transistors of the same type (nMOS or pMOS), the input-referred spectral noise density of the amplifier is

$$
\begin{aligned}
& V_{\mathrm{nf}, \mathrm{in}}^{2}(f) \\
& =\frac{K_{\mathrm{fn}}}{C_{\mathrm{ox}} f W_{1} L_{1}} \\
& \quad \times\left\{\begin{array}{l}
2\left[1+\frac{K_{\mathrm{fp}}}{K_{\mathrm{fn}}} \frac{\mu_{p}}{\mu_{n}}\left(\frac{L_{1}}{L_{2}}\right)^{2}\right] \\
+\frac{1}{\left(g_{m 3} R_{\mathrm{CM}}\right)^{2}}\left[\left(\frac{L_{1}}{L_{4}}\right)^{2}+\frac{K_{\mathrm{fp}}}{K_{\mathrm{fn}}} \frac{\mu_{p}}{\mu_{n}}\left(\frac{L_{1}}{L_{3}}\right)^{2}\right]+ \\
\frac{1}{\left(g_{m 3 P} R_{\mathrm{CM}}\right)^{2}}\left[\left(\frac{L_{1}}{L_{4 P}}\right)^{2}+\frac{K_{\mathrm{fp}}}{K_{\mathrm{fn}}} \frac{\mu_{p}}{\mu_{n}}\left(\frac{L_{1}}{L_{3 P}}\right)^{2}\right]
\end{array}\right\} .
\end{aligned}
$$

As the same $L$ was used for all the transistors $(L=180 \mathrm{~nm}$ in $130 \mathrm{~nm}$ technology), the flicker noise becomes

$$
\begin{aligned}
V_{\mathrm{nf}, \mathrm{in}}^{2}(f)=\frac{K_{\mathrm{fn}}}{C_{\mathrm{ox}} f W_{1} L}\left(1+\frac{K_{\mathrm{fp}}}{K_{\mathrm{fn}}} \frac{\mu_{p}}{\mu_{n}}\right) \\
\quad \times\left(2+\frac{1}{\left(g_{m 3} R_{\mathrm{CM}}\right)^{2}}+\frac{1}{\left(g_{m 3 P} R_{\mathrm{CM}}\right)^{2}}\right) .
\end{aligned}
$$

Note that for the moderately large $g_{m 3} R_{\mathrm{CM}}$ and $g_{m 3 \mathrm{P}} R_{\mathrm{CM}}$, the expression corresponds to the well-known flicker noise of a simple differential pair, as the first stage dominates noise contribution. Note also that, in these conditions, noise is dominated by the input pair $M_{1}$ and $M_{1 \mathrm{P}}$, as $K_{\mathrm{fn}}>K_{\mathrm{fp}}$ and $\mu_{n}>\mu_{p}$.

\section{Subthreshold Op-Amp}

The proposed op-amp can work in the subthreshold region with little modification. In the subthreshold region, quiescent power dissipation is lower as transistors can be biased with very low bias current (nAs) and with a supply voltage below $1 \mathrm{~V}$. In biomedical applications and wireless sensor networks, 


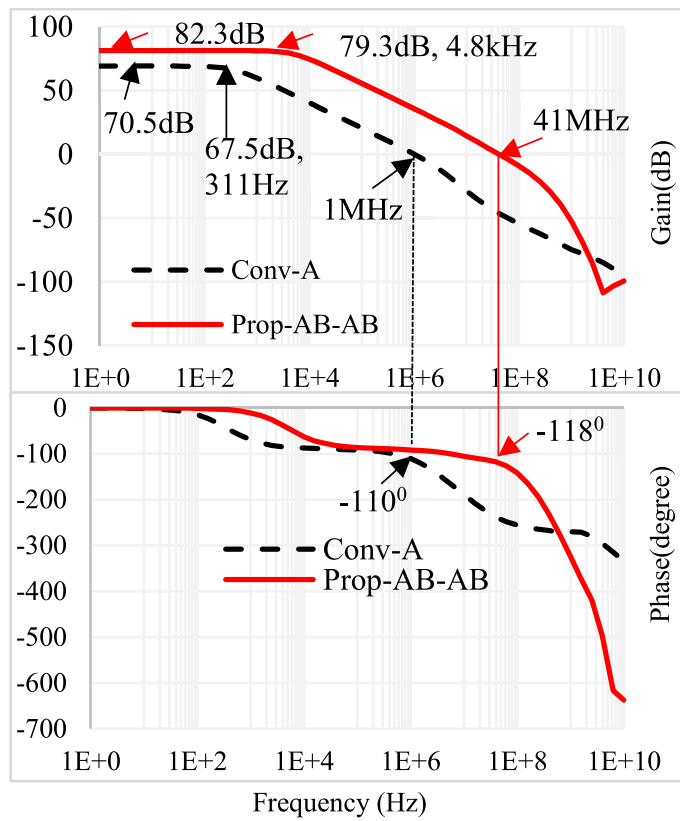

Fig. 10. Open-loop response of Conv-A and Proposed-AB-AB op-amp.

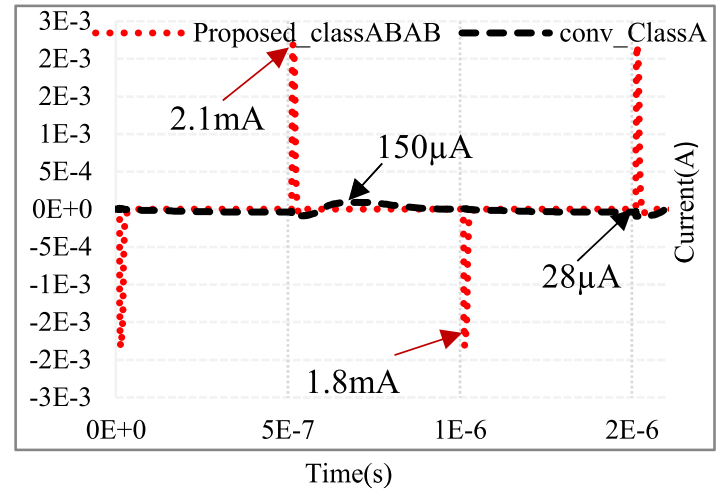

Fig. 11. Transient output currents of Conv-A and Proposed-AB-AB op-amp.

subthreshold op-amps are a good choice where high-speed is not a primary concern nevertheless a very low quiescent power dissipation is the foremost requirement.

Fig. 9(a) shows a conventional Miller op-amp (without telescopic input stage) working in the subthreshold region. Fig. 9(b) shows a modified version of the proposed op-amp for operation in subthreshold. To avoid body effect and reduce the supply requirements in subthreshold, the substrates of the two pMOS cascode transistors $\left(M_{3 \mathrm{CP}}\right.$ and $\left.M_{3 \mathrm{C}}\right)$ are connected to their source. In order to save the Silicon area, resistors used for local common mode feedback shown in Fig. 3 are replaced by MOS transistors operating in triode region with their gate connected to $V_{\mathrm{SS}}$. These transistors are designed to implement $1.5 \mathrm{M} \Omega$ resistors in order to provide again $A_{I}$ close to as in the strong-inversion case. In Section IV, simulation results of the op-amps in strong-inversion and in subthreshold regions are discussed.

\section{Simulation Results}

The Proposed-AB-AB and conventional op-amps were designed in 130-nm CMOS technology with nMOS and pMOS

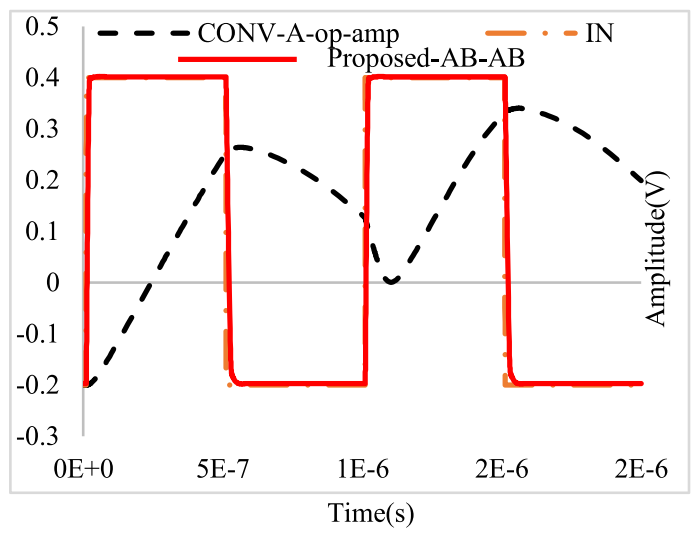

Fig. 12. Transient response of Proposed-AB-AB and Conv-A op-amp.

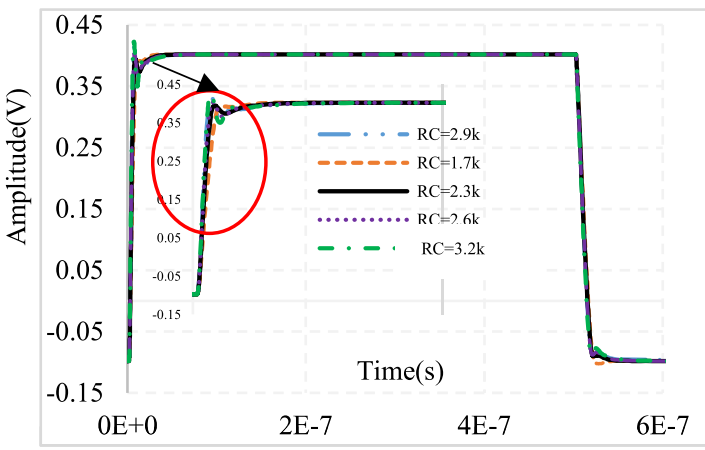

Fig. 13. Transient response of proposed op-amp for varying $\mathrm{R}_{C}$ in strong inversion.

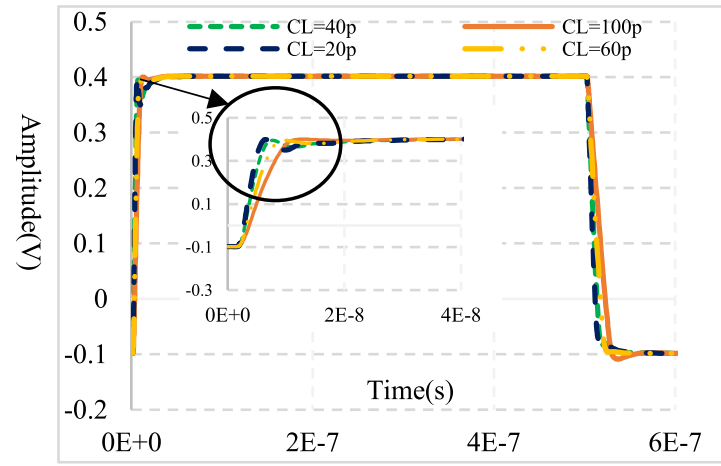

Fig. 14. Transient response for different $\mathrm{C}_{L}$ strong inversion.

unit transistor sizes $(W / L)_{N}=5 \mu \mathrm{m} / 0.18 \mu \mathrm{m}$ and $(W / L)_{P}=$ $20 \mu \mathrm{m} / 0.18 \mu \mathrm{m}$, respectively. They were simulated both with the same bias current and load capacitance $C_{L}=50 \mathrm{pF}$.

\section{A. Simulation Results in Strong Inversion}

The circuits of Figs. 1 and 3 were simulated with $\pm 0.6-\mathrm{V}$ dual supplies and bias current $I_{B}=14 \mu \mathrm{A}$. Fig. 10 shows its open-loop frequency response. It can be seen that the circuit of Fig. 1 (Conv-A) with telescopic input stage has a dc open-loop gain $A_{\mathrm{OLDC}}=70 \mathrm{~dB}$, a unity gain frequency $f_{U}=1 \mathrm{MHz}$, a 3 -dB frequency $f_{3 \mathrm{~dB}}=311 \mathrm{~Hz}$, and $\mathrm{GBW}=A_{\mathrm{OLDC}} f_{3 \mathrm{~dB}}=$ $1.1 \mathrm{MHz}$. 
TABLE II

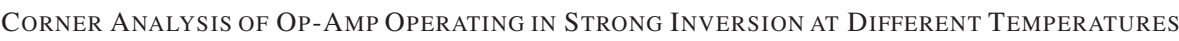

\begin{tabular}{|c|c|c|c|c|c|c|c|c|c|c|c|c|c|c|c|c|c|c|}
\hline \multicolumn{7}{|c|}{ At $\mathrm{T}=27^{0} \mathrm{C}$} & \multicolumn{6}{|c|}{ At $\mathrm{T}=100^{\circ} \mathrm{C}$} & \multicolumn{6}{|c|}{ At $\mathrm{T}=0^{\circ} \mathrm{C}$} \\
\hline Corner & $\mathrm{tt}$ & $\mathrm{ff}$ & fs & sf & ss & SD & $\mathrm{tt}$ & $\mathrm{ff}$ & fs & sf & ss & SD & $\mathrm{tt}$ & $\mathrm{ff}$ & fs & sf & ss & SD \\
\hline $\mathrm{I}_{\text {TotalQ }}(\mu \mathrm{A})$ & 158 & 163 & 156 & 161 & 156 & 3.1 & 154 & 159 & 150 & 156 & 149 & 4 & 162 & 166 & 158 & 164 & 157 & 3.8 \\
\hline THD (dB) & -58 & -59 & -54 & -55 & -45 & 5.5 & -59 & -62 & -62 & -60 & -55 & 2.88 & -48 & -60 & b54 & -42 & -40 & 8.3 \\
\hline GBW(MHz) & 63 & 68 & 61 & 63 & 60 & 3 & 43 & 46 & 41 & 42 & 39 & 2.5 & 77 & 82 & 73 & 77 & 82 & 3.8 \\
\hline PM & 61 & 60 & $60^{\circ}$ & $60^{\circ}$ & 58 & 1 & 59 & 58 & 58 & 59 & 58 & 1.3 & 60 & 59 & 58 & 61 & 58 & 1.3 \\
\hline Gain & 81 & 83 & 83 & 82 & 84 & 1 & 80 & 75 & 80 & 78 & 82 & 2.6 & 83 & 82 & 83 & 83 & 84 & 0.7 \\
\hline $\mathrm{SR}^{+}$ & 48 & 45 & 44 & 49 & 40 & 3.7 & 44 & 47 & 40 & 46 & 38 & 3.8 & 40 & 49 & 43 & 48 & 47 & 3.7 \\
\hline $\mathrm{SR}^{-}$ & 33 & 37 & 40 & 35 & 34 & 2.77 & 26 & 25 & 29 & 25 & 25 & 1.7 & 39 & 47 & 46 & 40 & 44 & 3.5 \\
\hline
\end{tabular}

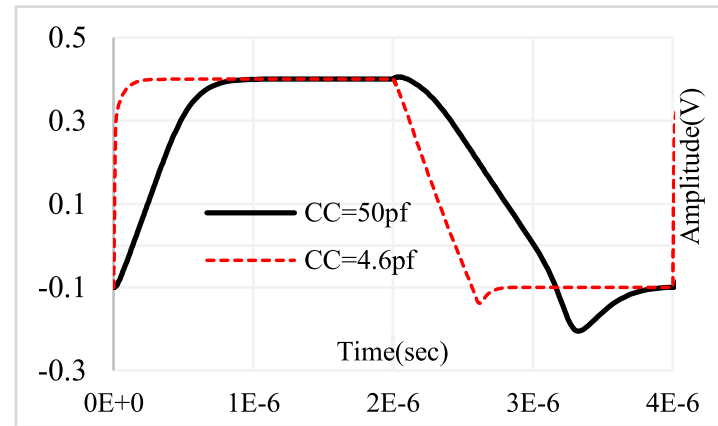

Fig. 15. Transient response shows settling time of Conv-A op-amp for $\mathrm{C}_{\mathrm{C}}=50 \mathrm{pF}$ and $\mathrm{C}_{\mathrm{C}}=4.6 \mathrm{pF}$ for $250-\mathrm{kHz}$ pulse in strong inversion.

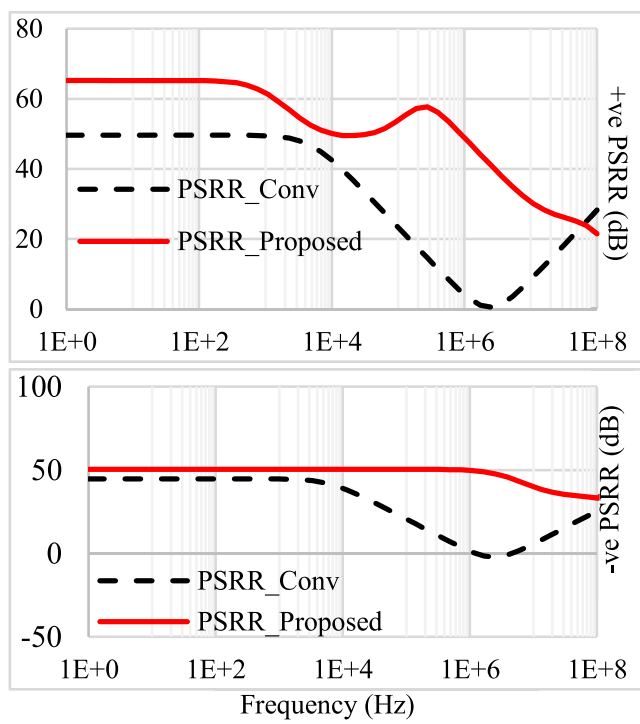

Fig. 16. Positive PSRR and negative PSRR in strong inversion.

The Proposed class AB-AB op-amp (Proposed-AB-AB) has $f_{U}=41 \mathrm{MHz}, A_{\mathrm{OLDC}}=82.3 \mathrm{~dB}, f_{3 \mathrm{~dB}}=4.8 \mathrm{kHz}$, and $\mathrm{GBW}$ $=63 \mathrm{MHz}$. Hence, the proposed op-amp can provide $13 \mathrm{~dB}$ higher open-loop gain, 63 times higher GBW, and 41 times higher $f_{U}$ at the expense of only 2.2 times increase in quiescent power dissipation compared to the conventional op-amp. Figs.11 and 12 show the transient responses of the Proposed$\mathrm{AB}-\mathrm{AB}$ and the Conv-A op-amp in voltage follower configuration with a $600 \mathrm{mVpp} 1-\mathrm{MHz}$ input square wave. From Figs. 11 and 12, it can be asserted that the Conv-A op-amp

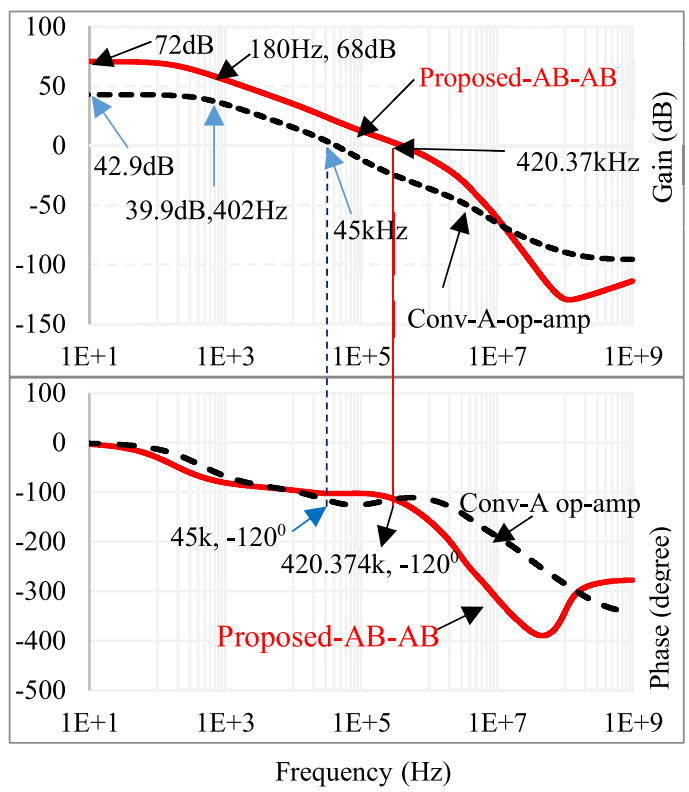

Fig. 17. Open-loop frequency response of op-amps in subthreshold.

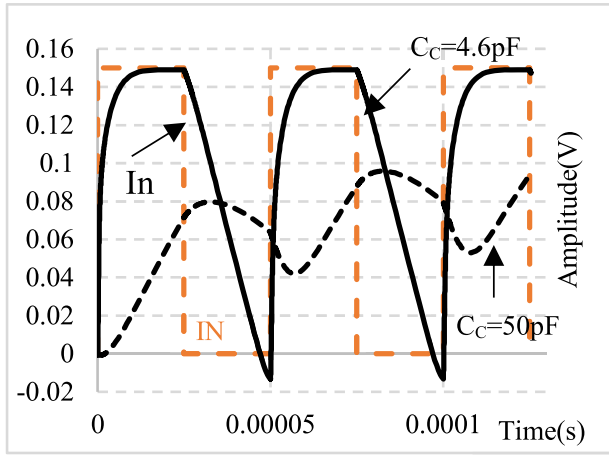

Fig. 18. Transient response of Conv-A op-amp for value of $\mathrm{C}_{\mathrm{C}}=4.6 \mathrm{pF}$ and $\mathrm{C}_{\mathrm{C}}=50 \mathrm{pF}$.

has much lower SR than the Proposed-AB-AB op-amp in both directions. The Conv-A op-amp delivers much lower positive and negative output peak currents $(150$ and $28 \mu \mathrm{A})$ than the Proposed-AB-AB op-amp (2.1 and $1.8 \mathrm{~mA})$ to the load capacitor $C_{L}$. Although conventional class A Miller op-amps with nMOS input stage can theoretically deliver large positive output currents (and negative output currents limited by the bias current of the output stage), the output current of the Conv-A op-amp in this example is limited in both directions by the SR of the internal node $X$. 
TABLE III

Corner Analysis of the Proposed Op-Amp Operating In SubThreshold At DifFERENT TEMPERATURES

\begin{tabular}{|c|c|c|c|c|c|c|c|c|c|c|c|c|c|c|c|c|c|c|}
\hline \multicolumn{7}{|c|}{ At $\mathrm{T}=27^{\circ} \mathrm{C}$} & \multicolumn{6}{|c|}{ At $\mathrm{T}=100^{\circ} \mathrm{C}$} & \multicolumn{6}{|c|}{ At $\mathrm{T}=0^{\circ} \mathrm{C}$} \\
\hline Corner & $\mathrm{tt}$ & $\mathrm{ff}$ & fs & sf & ss & SD & $\mathrm{tt}$ & ff & fs & sf & ss & SD & $\mathrm{tt}$ & $\mathrm{ff}$ & fs & $\mathrm{sf}$ & ss & SD \\
\hline $\mathrm{I}_{\text {TotalQ }}(\mu \mathrm{A})$ & 0.77 & $77 \mathrm{Th}$ & $\operatorname{sig} .71 / \pi$ & uth. 8 's & $\mathrm{roi}$ & 0.4 & that ha & bqe.4 & lishogd i & hip.4 & 1.1 han & & adego & soggsi & by.the & bbisger & $0.8 \mathrm{p}$ & lio:05 \\
\hline THD & -50 & $-49^{T h}$ & $=-1 i 4 \theta^{l v}$ & rsiqus of & eçyt is & aysizab & at 560 tp: & dx- $4 \theta^{i}$ & $y^{\prime}-19.5^{1}$ & $1043^{10}$ & $\Gamma$ & 19.29 & 3551 & -53 & -56 & -40 & -42 & 7 \\
\hline GBV & 716 & 700 & 720 & 700 & 723 & 16 & 632 & 600 & 614 & 630 & 600 & 16 & 532 & 495 & 492 & 486 & 490 & 18 \\
\hline PM & $62^{\circ}$ & $73^{\circ}$ & $57^{\circ}$ & $69^{\circ}$ & $59^{\circ}$ & 6 & $77^{\circ}$ & $93^{\circ}$ & $63^{\circ}$ & $88^{\circ}$ & $63^{0}$ & 13 & $65^{\circ}$ & $73^{\circ}$ & $68^{\circ}$ & $73^{\circ}$ & $66^{\circ}$ & 4 \\
\hline Gain & 71 & 66.4 & 72 & 67.2 & 72.4 & 2.8 & 46 & 32 & 53.6 & 36 & 57 & 10 & 80 & 69 & 62 & 63 & 61 & 7 \\
\hline $\mathrm{SR}^{+}$ & 0.10 & 0.08 & 0.11 & 0.10 & 0.11 & .01 & 0.07 & 0.07 & 0.08 & 0.06 & 0.09 & .01 & 0.10 & 0.09 & 0.09 & 0.09 & 0.04 & .02 \\
\hline SR & 0.13 & 0.12 & 0.08 & 0.18 & 0.73 & .04 & 0.10 & 0.06 & 0.12 & 0.07 & 0.15 & .04 & 0.05 & 0.18 & 0.03 & 0.15 & 0.02 & .07 \\
\hline
\end{tabular}

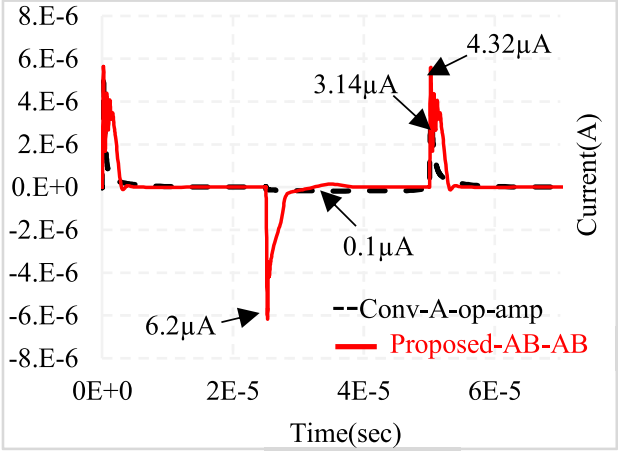

Fig. 19. Transient output current for op-amp in subthreshold.

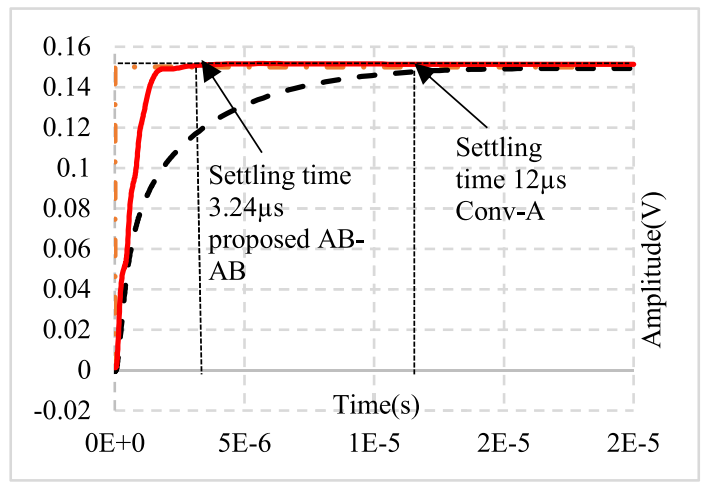

Fig. 20. Settling time of op-amps in subthreshold.

The class-A input stage of the Conv-A op-amp can deliver maximum positive and negative currents with value $2 I_{B}$ to the compensation capacitor $C_{C}$ at node $X$. The inclusion of a class- $\mathrm{AB}$ output stage does not lead to an improvement in the settling time as it is determined by the slew rate of the classA input stage at node X. In addition, the much lower GBW value of the Conv-A op-amp also results in long exponential settling times in both directions. From Figs. 11 and 12, it can be observed that the inclusion of class-AB input and output stages enhances the dynamic output current in the ProposedAB-AB op-amp. The Proposed-AB-AB op-amp has positive and negative output current enhancement factors 14 and 63 , compared to the conventional op-amp. These results satisfy the theory of the proposed op-amp described in the previous section. To observe the effect of pole-zero doublet mismatches in the settling time Fig. 13 shows a transient response for $R_{C}$ in the range from 1.7 to $3.2 \mathrm{k} \Omega$. The $0.1 \% t_{\text {Setl }}$ of the

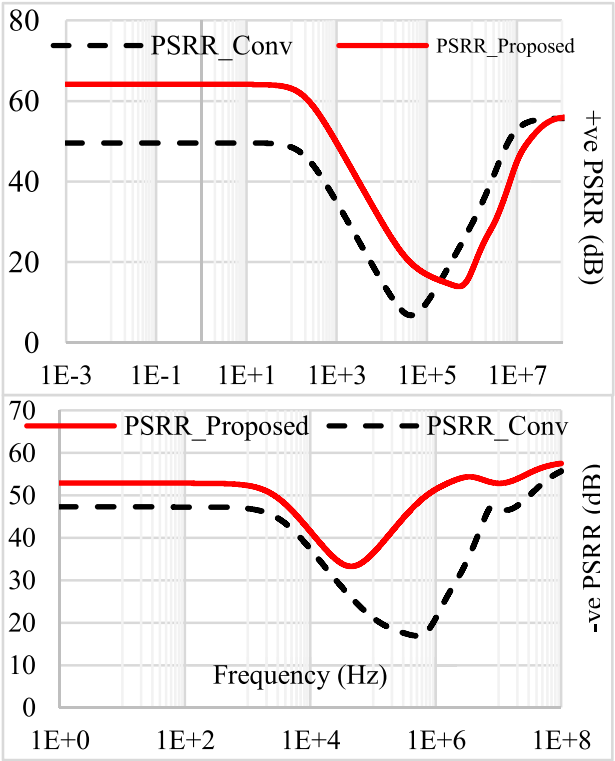

Fig. 21. Positive and negative PSRR of the Proposed-AB-AB Op-amp.

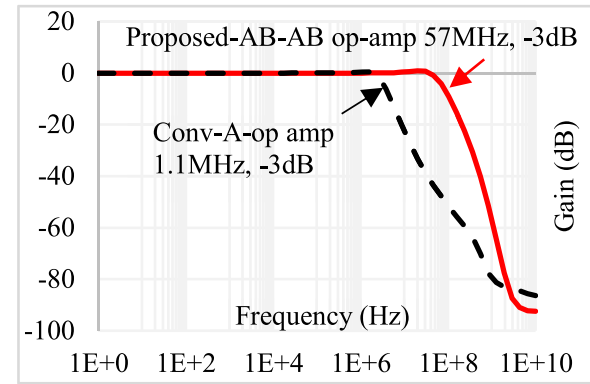

Fig. 22. Experimental frequency response of op-amps (strong inversion) in voltage follower configuration.

op-amp is measured considering the time required for the response to reach and stay within a range of $\pm 0.1 \%$ of the final value [13]. For a 1-MHZ $500 \mathrm{mV}$ peak-to-peak amplitude step input the $0.1 \%$ positive settling time $t_{\text {Setl }}^{+}$varies from 28 to $66 \mathrm{~ns}$ and the negative settling time $t_{\text {Setl }}^{-}$varies from 35 to $70 \mathrm{~ns}$ for $f_{\mathrm{z}} / f_{\mathrm{p}}$ mismatch factor within the range 1.67-0.8. Fig. 14 shows the transient response of the proposed op-amp for different $C_{L}$ values that lead to a pole-zero mismatch factor within the range of 0.4-2.4. In this case, the $0.1 \%$ $t_{\text {Setl }}^{+}$varies from 52 to $35 \mathrm{~ns}$ and $0.1 \% t_{\text {Setl }}^{-}$varies from 68 to $60 \mathrm{~ns}$ for load capacitances within the range $20 \mathrm{pF}<C_{L}<$ $100 \mathrm{pF}$. An ideal single pole op-amp has a $0.1 \%$ settling time 


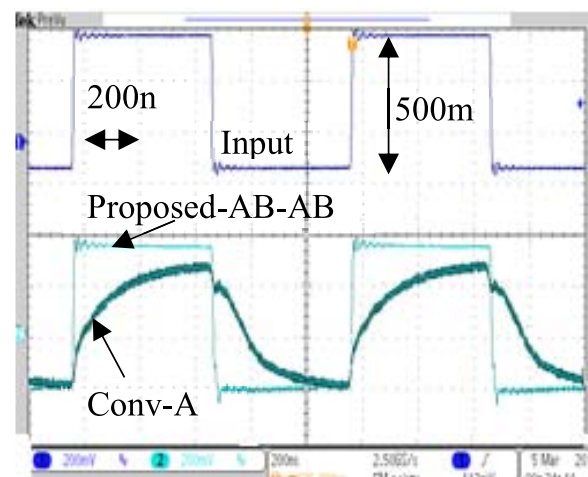

Fig. 23. Experimental transient response of the proposed op-amp at stronginversion for $1-\mathrm{MHz} 500-\mathrm{mV}$ input amplitude pulse.

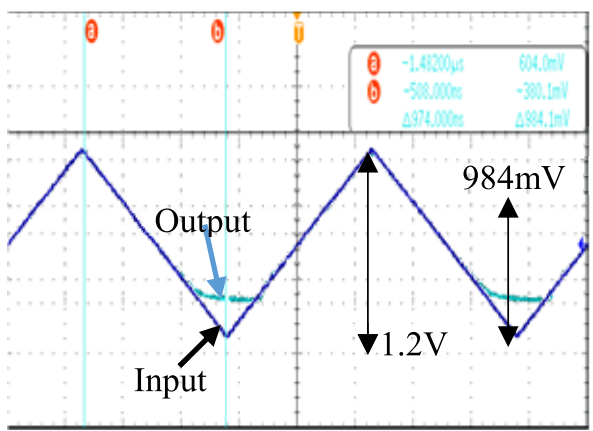

Fig. 24. Experimental input range for the Proposed-AB-AB op-amp for $\pm 600 \mathrm{mV} 1-\mathrm{MHz}$ triangular pulse at strong inversion.

$t_{\text {setl }}=6.9 /(2 \pi \mathrm{GBW})$ which for GBW $=63 \mathrm{MHz}$ results in $t_{\text {setl }}=17.3 \mathrm{~ns}$. The $0.1 \%$ settling times of the proposed op-amp are factors 2-4 larger than for an ideal single pole op-amp but essentially lower than for the conventional op-amp. Fig. 15 shows the settling time of the Conv-A op-amp for $C_{C}=4.6 \mathrm{pF}\left(t_{\text {Setl }}^{+}=285 \mathrm{~ns}, t_{\text {Setl }}^{-}=890 \mathrm{~ns}\right)$ and $C_{C}=50 \mathrm{pF}$ $\left(t_{\text {Setl }}^{+}=1.2 \mu \mathrm{s}\right.$ and $\left.t_{\text {Setl }}^{-}=2 \mu \mathrm{s}\right)$.

Fig. 16 shows the positive and negative PSRR of the Proposed-AB-AB and Conv-A op-amp. The Proposed-AB-AB op-amp provides 67 - and $50-\mathrm{dB}$ positive and negative PSRR. The positive PSRR is $17 \mathrm{~dB}$ higher than the conventional op-amp and remains high over a wider range of frequencies. This is attributed to the higher dc gain and BW. To show the robustness of the proposed op-amp against process and temperature variations, corner analysis at three different temperatures for important performance parameters of the circuit are given in Table II. It can be asserted that proposed op-amp is robust against variation of process and temperature and can provide wide GBW. Standard deviation (SD) is shown in the table for each parameter for variation of the process for considered temperatures.

\section{B. Simulation Results in Subthreshold Region}

The proposed and conventional op-amps were also simulated in subthreshold with $\pm 0.25-\mathrm{V}$ supply voltage and bias current $I_{B}=70 \mathrm{nA}$. Fig. 17 shows the frequency response of the conventional and proposed op-amps. The Proposed-AB$\mathrm{AB}$ op-amp has 72-dB open-loop dc gain and unity gain frequency $f_{U}=420.37 \mathrm{kHz}$ with $60^{\circ}$ phase margin. The Conv-A

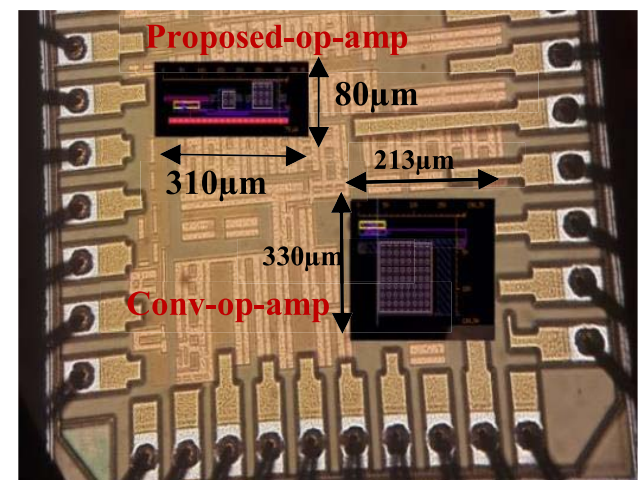

Fig. 25. Micrograph of the Proposed-AB-AB and Conv-A op-amp in strong inversion.

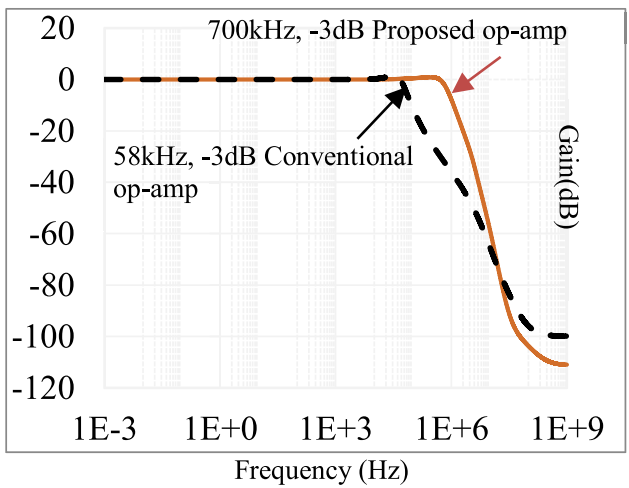

Fig. 26. Experimental frequency response of the Proposed-AB-AB and Conv-A op-amp at subthreshold.

op-amp has 42.9-dB open-loop dc gain, unity gain frequency $f_{U}=45 \mathrm{kHz}$ and $60^{\circ}$ phase margin. Fig. 18 shows that the Conv-A op-amp suffers from a poor settling time for the larger compensation capacitor. The Conv-A op-amp in subthreshold was fabricated with $C_{C}=4.6 \mathrm{pF}$. Fig. 19 shows the transient output current of the proposed and conventional op-amps in subthreshold. The Proposed-AB-AB op-amp can provide maximum positive and negative output currents of 4.32 and $6.24 \mu \mathrm{A}$, respectively. Hence, the negative current limitation is significantly improved in the Proposed-AB-AB op-amp. The positive setting times of the Proposed-AB-AB and ConvA op-amps are 3.2 , and $12 \mu \mathrm{s}$, respectively, according to Fig. 20. The negative settling times of the Proposed-AB$\mathrm{AB}$ and Conv-A-op-amps are 13 and $197 \mu$ s, respectively. Positive and negative PSRRs are 64 and $53 \mathrm{~dB}$, respectively, whereas conventional op-amp has 50- and $47-\mathrm{dB}$ positive and negative PSRRs. Fig. 21 shows the PSRR responses of the op-amps. Corner analysis of the proposed op-amp at different temperatures operating in the subthreshold region is given in Table III. It can be observed that the proposed op-amp is stable against the variation of process and temperature. The SD of each parameter for variation of the process has been given in Table III for the considered temperatures $\left(0{ }^{\circ} \mathrm{C}, 27{ }^{\circ} \mathrm{C}\right.$, $\left.100{ }^{\circ} \mathrm{C}\right)$.

\section{EXPERIMENTAL RESUlts}

Test chip prototypes of the proposed op-amps with $C_{C}=$ $4.6 \mathrm{pF}$ and conventional op-amps with $C_{C}=50 \mathrm{pF}$ for strong inversion were fabricated in $130-\mathrm{nm}$ CMOS process 
TABLE IV

Summary of the Measurement Results and Performance Comparison

\begin{tabular}{|c|c|c|c|c|c|c|c|c|c|c|c|c|}
\hline Parameter(units) & Conv & & This v & ork & [14] & [15] & [9] & [16] & [17] & [18] & [4] & [19] \\
\hline Inversion level & SI & SBT & SI & SBT & SI & SI & SI & SBT & SI & SI & SBT & SBT \\
\hline Supply voltage (V) & \pm 0.6 & \pm 0.25 & \pm 0.6 & \pm 0.25 & 1.8 & \pm 1 & \pm 1.65 & 0.7 & \pm 1.65 & \pm 0.9 & 1 & 0.25 \\
\hline $\mathrm{SR}+(\mathrm{V} / \mu \mathrm{s})$ & 1.5 & .07 & 37 & .08 & 74.1 & 9.8 & 88.2 & 1.8 & 24 & 31 & .007 & .0006 \\
\hline SR- $(\mathrm{V} / \mu \mathrm{s})$ & 0.3 & .002 & 34 & .12 & NA & 7.6 & 42 & 3.8 & 22 & 28 & .003 & .0008 \\
\hline DC gain $(\mathrm{dB})$ & 70 & 43 & 83 & 72 & 72 & 81.7 & 82 & 57.5 & 68 & 90.8 & 120 & 60 \\
\hline $\operatorname{PM}\left({ }^{\circ}\right)$ & 70 & 60 & 62 & 60 & 50 & 60 & NA & 60 & 73 & 58.4 & 54 & 52.5 \\
\hline PSRR+@DC (dB) & 49 & 49 & 67 & 64 & NA & 72 & 95 & 52.1 & 78 & 64 & 98 & NA \\
\hline PSRR-@DC (dB) & 45 & 47 & 50 & 53 & NA & 74 & 83 & 66.4 & 75 & 66 & NA & NA \\
\hline $\begin{array}{l}\text { Eq.input-noise } \\
(\mathrm{nV} / \sqrt{ } \mathrm{Hz})\end{array}$ & $\begin{array}{l}68 @ .1 \\
M\end{array}$ & $\begin{array}{r}220 \\
@ 10 \mathrm{k}\end{array}$ & $\begin{array}{l}25 @ \\
1 \mathrm{M}\end{array}$ & $\begin{array}{r}160 \\
@ .1 \mathrm{M}\end{array}$ & $\begin{array}{r}0.8 \\
\text { a. } 1 \mathrm{M}\end{array}$ & $\begin{array}{l}35 @ \\
1 \mathrm{M}\end{array}$ & NA & $\begin{array}{c}100 \\
@ 1 \mathrm{M}\end{array}$ & NA & 27 & $\begin{array}{r}4850 \\
@ 1 \mathrm{kHz}\end{array}$ & 3300 \\
\hline $\mathrm{I}_{\text {TotalQ }}(\mu \mathrm{A})$ & 70 & 0.3 & 158 & .78 & 6611 & 60 & 82 & 36.3 & 418 & 43 & .195 & .072 \\
\hline Power $(\mu \mathrm{W})$ & 84 & 0.15 & 190 & .39 & 11900 & 120 & 270 & 25.4 & 1380 & 80 & .195 & .018 \\
\hline $\mathrm{AFOM}_{\mathrm{L}}$ & 2.5 & 67 & 447 & 512 & 18 & 222 & 155 & 71 & 16 & 438 & 769 & 6 \\
\hline $\mathrm{AFOM}_{\mathrm{S}}$ & 0.9 & 1933 & 828 & 4651 & 21 & 138 & NA & 118 & 16 & 195 & 5128 & 19 \\
\hline $\mathrm{IFOM}_{\mathrm{L}}$ & .2 & 0.3 & 11 & 5 & 2 & 9 & 10 & 1 & 2 & 16 & 3 & 0.1 \\
\hline $\mathrm{IFOM}_{\mathrm{S}}$ & 0.8 & 10 & 20 & 46 & 3 & 5 & NA & 2 & 2 & 7 & 20 & 0.4 \\
\hline
\end{tabular}

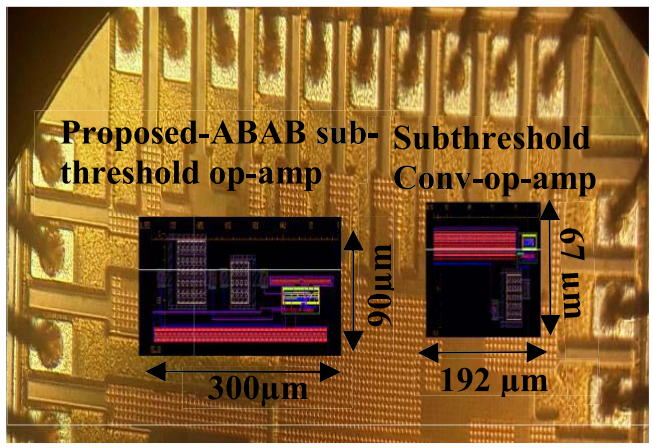

Fig. 27. Micrograph of the Proposed-AB-AB and Conv-A op-amp in subthreshold.

technology. For subthreshold operation, the proposed and Conv-A op-amps were fabricated with $C_{C}=4.6 \mathrm{pF}$ in $130-\mathrm{nm}$ CMOS process also. The chips were tested with \pm 0.6 -Vsupply voltage, quiescent current $I_{\text {Total } Q}=158 \mu \mathrm{A}$ in strong inversion, $\pm 0.25-\mathrm{V}$ supply voltage and total quiescent current $0.78 \mu \mathrm{A}$ in the subthreshold region. In both cases, the load capacitance $C_{L}$ is $50 \mathrm{pF}$.

\section{A. Operation in Strong Inversion}

The measured frequency response of the Conv-A and Proposed-AB-AB op-amps as voltage followers are shown in Fig. 22. The Proposed-AB-AB op-amp has a bandwidth of $57 \mathrm{MHz}$, whereas the Conv-A op-amp of Fig. 1 has a bandwidth of $1.1 \mathrm{MHz}$. Note that the bandwidth of the op-amp is improved by a factor of 52 compared to the conventional one. Transient responses of the op-amps are shown in Fig. 23 to

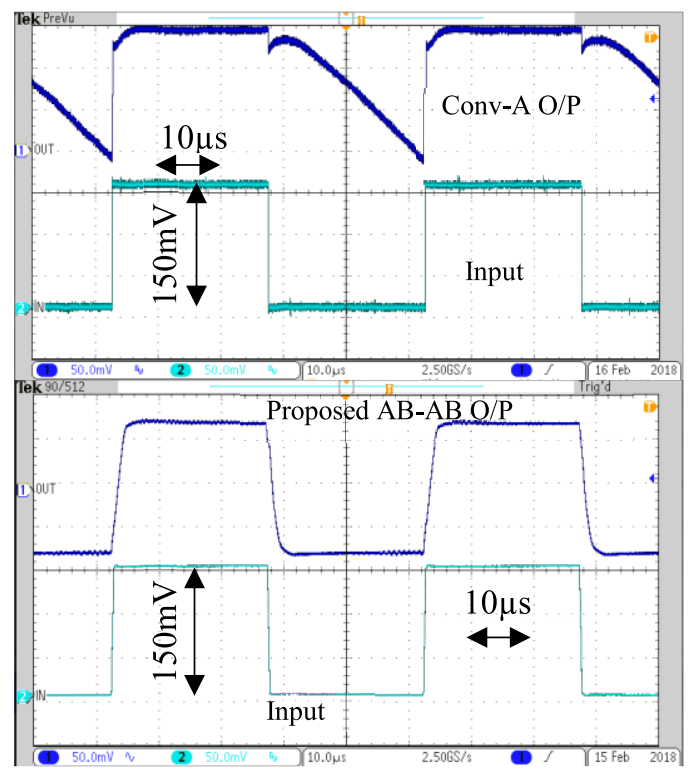

Fig. 28. Experimental transient response of the Proposed-AB-AB and Conv-A op-amp for $20-\mathrm{kHz}$ pulse of $150-\mathrm{mV}$ amplitude at subthreshold.

a 1-MHz, 500-mV pulse input waveform. The positive SRs of the Proposed-AB-AB and Conv-A op-amp are 37 and $1.5 \mathrm{~V} / \mu \mathrm{s}$, respectively. The negative $\mathrm{SRs}$ are 34 and $0.3 \mathrm{~V} / \mu \mathrm{s}$ for the Proposed-AB-AB and Conv-A op-amps. Thus, the SR of the proposed op-amp is 113 times higher than for the Conv-A op-amp obtained from the experimental results. The $\mathrm{SR}$ of the op-amp is given by $\mathrm{SR}=I_{\text {OutMax }} /\left(C_{L}+C_{C}\right)$. From this expression of SR experimental maximum positive and negative current can be calculated. 


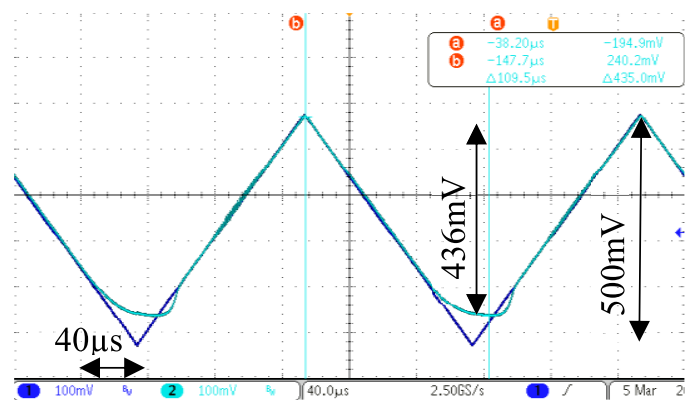

Fig. 29. Experimental input range for the Proposed-AB-AB op-amp at subthreshold for $\pm 250 \mathrm{mV}, 5-\mathrm{kHz}$ triangular pulse.

Experimental maximum positive output currents for the Conv-A and Proposed-AB-AB op-amp are $150 \mu \mathrm{A}$ and $2 \mathrm{~mA}$. The maximum negative currents for Conv-A and Proposed$\mathrm{AB}-\mathrm{AB}$ op-amps are $30 \mu \mathrm{A}$ and $1.8 \mathrm{~mA}$. Hence, current efficiency $\mathrm{CE}$ of the Proposed-AB-AB op-amp is 11 , whereas for Conv-A it is only 0.4. The input/output common mode ranges of the proposed op-amp are shown in Fig. 24 for a $\pm 600-\mathrm{mV}, 1-\mathrm{MHz}$ triangular input waveform. They have values of +600 and $-384 \mathrm{mV}$ for the proposed op-amp. Fig. 25 shows the chip micrograph of the proposed and conventional op-amps. Designed layouts are superimposed due to the opaque passivation layer. The silicon area consumed by the conventional op-amp is $0.07 \mathrm{~mm}^{2}$, whereas the area of the proposed op-amp is only $0.02 \mathrm{~mm}^{2}$. Hence, in the proposed approach, higher large- and small-signal FOM can be obtained, while the silicon area can be essentially reduced because of smaller $C_{C}$.

\section{B. Operation in Subthreshold Region}

Fig. 26 shows the experimental frequency response of the Proposed-AB-AB and Conv-A op-amp in voltage follower configuration. The bandwidths of the Proposed-AB$\mathrm{AB}$ and Conv- A op-amp are 700 and $58 \mathrm{kHz}$, respectively. Fig. 27 shows the micrograph of the fabricated chip with the op-amps designed for subthreshold operation. The area occupied by the Proposed-AB-AB op-amp is $0.02 \mathrm{~mm}^{2}$ which is two times more than the Conv-A op-amp designed in subthreshold. Though the proposed and conventional class-A op-amps are using similar compensation capacitor, the former occupied a larger area because the Proposed AB-AB opamp has an extra capacitor $C_{\mathrm{BAT}}$. The transient responses of conventional and proposed op-amps are given in Fig. 28 for a $20-\mathrm{kHz}$ square waveform with $150-\mathrm{mV}$ amplitude. The positive and negative SRs of the Proposed-AB-AB op-amp are 0.08 and $0.12 \mathrm{~V} / \mu \mathrm{s}$, whereas for Conv-A, they are 0.07 and $0.002 \mathrm{~V} / \mu \mathrm{s}$, respectively.

Hence, the negative SR of the Conv-A op-amp is very poor. The maximum positive and negative output currents for the Proposed-AB-AB op-amp are 4.2 and $6.24 \mu \mathrm{A}$, while the Conv-A op-amp has maximum positive and negative current of 3.6 and $0.1 \mu \mathrm{A}$. The $\mathrm{CE}$ in subthreshold for the Proposed $\mathrm{AB}-\mathrm{AB}$ op-amp is 6 and for Conv-A, it is 0.3. Hence, the proposed op-amp can achieve improved negative SR. Finally, the proposed op-amp can provide 12 times higher GBW, 62 times higher negative output current at the expense of increasing the quiescent power dissipation by a factor 2.6. The input/output common mode ranges for the Proposed-AB-AB op-amp are between 242 and $-194.9 \mathrm{mV}$ for $\pm 250-\mathrm{mV} 5-\mathrm{kHz}$ triangular pulse, as shown in Fig. 29.

A comprehensive comparison of the proposed op-amp's performance with other state-of-the-art class-AB amplifiers is given in Table IV. From the table, it can be asserted that except the $\mathrm{Si}$ area related FOMs (strongly technology dependent), the Proposed-AB-AB op-amp has the highest small-signal, largesignal, and global FOM in strong inversion and subthreshold region. However, in strong inversion [18], it has 1.4 times higher $\mathrm{IFOM}_{L}$; all other FOMs of it are lower.

\section{CONCLUSION}

A power-efficient Miller op-amp architecture was reported and experimentally verified. Two versions of the op-amp were presented here: working in strong inversion as well as in subthreshold with little modification. These two versions were fabricated in 130-nm CMOS technology.

Experimental results verified that the proposed op-amp working with $1.2-\mathrm{V}$ supply voltage has essentially improved large- and small-signal $\mathrm{FOM}_{\mathrm{LS}}=9$ and $\mathrm{FOM}_{\mathrm{SS}}=17$. The proposed op-amp operating in subthreshold also has the largest FOMs: FOM $\mathrm{LS}$ and $\mathrm{FOM}_{\mathrm{SS}}$ of 10 and 92, respectively. According to the literature presented in this paper, consequently, the highest value of the global FOM can be achieved which determines the ultimate speed of the proposed op-amp.

As in the subthreshold region, the op-amp can also operate with a very low supply voltage $( \pm 0.25 \mathrm{~V})$; it can be used in applications where power constraints exist, such as in biomedical instruments and wireless sensor networks. Finally, it can be asserted that experimental results validated the proposed circuit's principle.

\section{REFERENCES}

[1] G. Giustolisi, G. Palmisano, and T. Segreto, "1.2-V CMOS op-amp with a dynamically biased output stage," IEEE J. Solid-State Circuits, vol. 35, no. 4, pp. 632-636, Apr. 2000.

[2] K. J. de Langen and J. H. Huijsing, "Compact low-voltage powerefficient operational amplifier cells for VLSI," IEEE J. Solid-State Circuits, vol. 33, no. 10, pp. 1482-1496, Oct. 1998.

[3] H. F. Achigui, C. J. B. Fayomi, and M. Sawan, "1-V DTMOS-based class-AB operational amplifier: Implementation and experimental results," IEEE J. Solid-State Circuits, vol. 41, no. 11, pp. 2440-2448, Nov. 2006.

[4] A. D. Grasso, D. Marano, G. Palumbo, and S. Pennisi, "Design methodology of subthreshold three-stage CMOS OTAs suitable for ultralow-power low-area and high driving capability," IEEE Trans. Circuits Syst. I, Reg. Papers, vol. 62, no. 6, pp. 1453-1462, Jun. 2015.

[5] A. Paul, J. Ramírez-Angulo, and A. Torralba, "Bandwidth-enhanced high current efficiency class-AB buffer with very low output resistance," IEEE Trans. Circuits Syst. II, Exp. Briefs, vol. 65, no. 11, pp. 1544-1548, Nov. 2018.

[6] R. J. Baker, CMOS: Circuit Design, Layout and Simulation. Hoboken, NJ, USA: Wiley, 2011.

[7] J. Ramirez-Angulo, R. G. Carvajal, J. A. Galan, and A. Lopez-Martin, "A free but efficient low-voltage class-AB twostage operational amplifier," IEEE Trans. Circuits Syst. I, Reg. Papers, vol. 53, no. 7, pp. 568-571, Jul. 2006.

[8] J. Ramirez-Angulo and M. Holmes, "Simple technique using local CMFB to enhance slew rate and bandwidth of one-stage CMOS opamps," Electron. Lett., vol. 38, no. 23, pp. 1409-1411, Nov. 2002. 
[9] A. Roman-Loera, J. Ramirez-Angulo, A. Lopez-Martin, and R. G. Carvajal, "Free class AB-AB Miller opamp with high current enhancement," Electron. Lett., vol. 51, no. 3, pp. 215-217, 2015.

[10] A. A. Hamoui and N. C. Rumin, "An analytical current, delay, and power model for the submicron CMOS inverter," in Proc. IEEE 6th Int. Conf. Electron., Circuits Syst. (ICECS), vol. 3, Sep. 1999, pp. 1547-1551.

[11] R. E. Schreier and T. Caldwell. Amplifier Design. Accessed: Sep. 4, 2019. [Online]. Available: http://individual.utoronto.ca/schreier/ ece1371.html

[12] B. Razavi, Design of Analog CMOS Integrated Circuits, 2nd ed. New York, NY, USA: McGraw-Hill, 2000.

[13] C. T. Chuang, "Analysis of the settling behavior of an operational amplifier," IEEE J. Solid-State Circuits, vol. SSC-17, no. 1, pp. 74-80, Feb. 1982.

[14] S. Sutula, M. Dei, L. Terés, and F. Serra-Graells, "Variable-mirror amplifier: A new family of process-independent class-AB single-stage OTAs for low-power SC circuits," IEEE Trans. Circuits Syst. I, Reg. Papers, vol. 63, no. 8, pp. 1101-1110, Aug. 2016.

[15] M. P. Garde, A. Lopez-Martin, R. G. Carvajal, and J. Ramírez-Angulo, "Folded cascode OTA with $5540 \mathrm{MHzpF} / \mathrm{mA}$ FoM," in Proc. IEEE Int. Symp. Circuits Syst. (ISCAS), May 2018, pp. 1-5.

[16] E. Cabrera-Bernal, S. Pennisi, A. D. Grasso, A. Torralba, and R. G. Carvajal, "0.7-V three-stage class-AB CMOS operational transconductance amplifier," IEEE Trans. Circuits Syst. I, Reg. Papers, vol. 63 , no. 11, pp. 1807-1815, Nov. 2016

[17] J. Aguado-Ruiz, A. Lopez-Martin, J. Lopez-Lemus, and J. Ramirez-Angulo, "Power efficient class AB op-amps with high and symmetrical slew rate," IEEE Trans. Very Large Scale Integr. (VLSI) Syst., vol. 22, no. 4, pp. 943-947, Apr. 2014.

[18] S. Pourashraf, J. Ramirez-Angulo, A. J. Lopez-Martin, and R. Gonzalenz-Carvajal, "A highly efficient composite class- $\mathrm{AB}-\mathrm{AB}$ miller op-amp with high gain and stable from $15 \mathrm{pF}$ up to very large capacitive loads," IEEE Trans. Very Large Scale Integr. (VLSI) Syst., vol. 26, no. 10, pp. 2061-2072, Oct. 2018.

[19] L. H. C. Ferreira and S. R. Sonkusale, "A 60-dB gain OTA operating at 0.25 -V power supply in 130-nm digital CMOS process," IEEE Trans. Circuits Syst. I, Reg. Papers, vol. 61, no. 6, pp. 1609-1617, Jun. 2014.

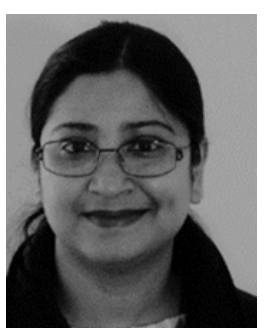

Anindita Paul (M'16) was born in Chinsurah, India. She received the M.S.E.E degree in VLSI design from the Institute of Radio Physics and Electronics, University of Calcutta, Kolkata, India. She is currently working toward the Ph.D. degree at the Klipsch School of Electrical Engineering, New Mexico State University, Las Cruces, NM, USA.

Her current research interests include low-voltage low-power analog circuit design.

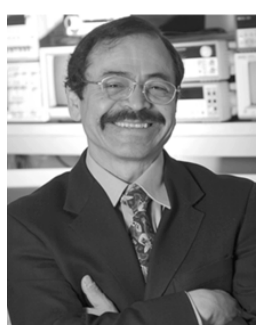

Jaime Ramírez-Angulo (M'82-F'00) received the B.Sc. and M.S.E.E. degrees from the National Polytechnic Institute, Mexico City, Mexico, in 1974 and 1976, respectively, and the Ph.D. degree from the University of Stuttgart, Stuttgart, Germany, in 1982.

He was a Professor with the National Institute for Astrophysics Optics and Electronics, Mexico, and Texas A\&M University, College Station, TX, USA. $\mathrm{He}$ is currently a Distinguished Award Professor with the Klipsch School of Electrical and Computer Engineering, New Mexico State University, Las Cruces, NM, USA. His current research interests include the design and test of analog and mixed-signal very large-scale integrated circuits.

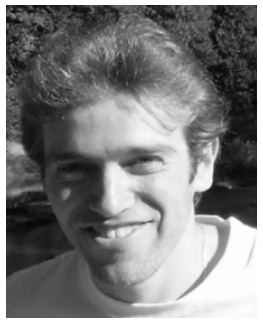

Antonio J. López-Martín (M’04-SM'11) received the M.S. and Ph.D. degrees (Hons.) from the Public University of Navarra, Pamplona, Spain, in 1995 and 1999, respectively.

He was a Visiting Professor at the New Mexico State University, Las Cruces, NM, USA. He is currently a Professor with the Public University of Navarra. He has authored or coauthored over 400 technical contributions in books, journals, and conferences. He holds six international patents. His current research interests include wireless transceivers and sensor interfaces with emphasis on low-voltage low-power implementations.

Dr. López-Martín was a recipient of the Talgo Technological Innovation Award in 2012, the Caja Navarra Research Award in 2007, the Young Investigator Award from the Completeness University of Madrid in 2006, the 2005 IEEE Transactions on Education Best Paper Award, and the European Center of Industry and Innovation Award in 2004 for excellence in transfer of research results to industry. He was an Associate Editor of the IEEE TRANSACTIONS ON CIRCUITS AND SYSTEMS-PART II: EXPRESS BRIEFS from 2006 to 2007 and the IEEE TRANSACTION ON CIRCUITS AND SYSTEMS-PART I: Regular PAPERS from 2008 to 2009.

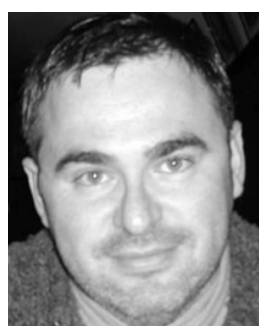

Ramón Gonzalez Carvajal (M'99-SM'04) received the M.Sc. degree in electrical engineering and the Ph.D. degree (Hons.) from the University of Seville, Seville, Spain, in 1995 and 1999, respectively.

In 1996, he joined the Department of Electronic Engineering, School of Engineering, University of Seville, as an Associate Professor, where he became a Professor in 2002. In 1999, he joined the Klipsch School of Electrical Engineering, Las Cruces, NM, USA. He has authored or coauthored over 60 papers in international journals and authored 200 papers in international conferences. His current research interests include low-voltage low-power analog circuit design, A/D and D/A conversion, and analog and mixed-signal processing.

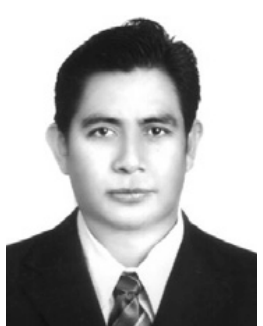

José Miguel Rocha-Pérez (M'16) received the M.Sc. and Ph.D. degrees from the Instituto Nacional de Astrofísica, Optica y Electrónica (INAOE), Puebla, Mexico, in 1991 and 1999, respectively.

He was a Visiting Scholar at the Department of Electrical Engineering, Texas A\&M University, College Station, TX, USA, in 2002, and CINVESTAV Guadalajara Mexico, Zapopan, Mexico, in 2003. $\mathrm{He}$ is currently with the Electronics Department, INAOE. His current research interests include lowvoltage, low-power integrated circuits design for biomedical applications. 